\title{
GENERALIZED BSDE DRIVEN BY A LÉVY PROCESS
}

\author{
MOHAMED EL OTMANI
}

Received 4 March 2005; Accepted 14 June 2006

We study the solution of one-dimensional generalized backward stochastic differential equation driven by Teugels martingales and an independent Brownian motion. We prove existence and uniqueness of the solution when the coefficient verifies some conditions of Lipschitz. If the coefficient is left continuous, increasing, and bounded, we prove the existence of a solution.

Copyright (C) 2006 Mohamed El Otmani. This is an open access article distributed under the Creative Commons Attribution License, which permits unrestricted use, distribution, and reproduction in any medium, provided the original work is properly cited.

\section{Introduction}

A linear version of backward stochastic differential equations (BSDEs) was first studied by Bismut [4] as the adjoint processes in the maximum principal of stochastic control. Pardoux and Peng in [20] introduced the notion of nonlinear BSDE. Since then, the interest in BSDEs has increased.

Indeed, BSDEs provide connection with mathematical finance [10], stochastic control [11], and stochastic game [9]. On the other hand, this class of BSDEs is a powerful tool to give probabilistic formulas for solution of partial differential equations (see $[18,19])$.

Given a Brownian motion $\left(W_{t}\right)_{0 \leq t \leq T}$, we denote by $\left(\mathscr{F}_{t}\right)_{0 \leq t \leq T}$ its natural filtration. Consider the nonlinear BSDE:

$$
Y_{t}=\xi+\int_{t}^{T} f\left(s, Y_{s}, Z_{s}\right) d s-\int_{t}^{T} Z_{s} d W_{s}
$$

where $\xi$ is an $\mathscr{F}_{T}$-measurable random variable that will become certain only at the terminal time $T$, and $f$ is a progressively measurable process.

In [20], the authors showed that there exists a unique $\mathscr{F}_{t}$-adapted process $(Y, Z)$ solution of the BSDE (1.1), when the coefficient $f$ is Lipschitz in $y$ and $z$, $\xi$ is square integrable. 
Many existence and uniqueness results have been proved in relaxing the Lipschitz condition of the coefficient. For instance, Peng introduced for the first time monotone coefficient in [22], see also $[2,6,8]$. In the one-dimensional case, Lepeltier and San Martin [13] described the BSDEs with a continuous coefficient, and Kobylanski [12] studied those with a coefficient which is quadratic in $z$.

Further, other settings of BSDEs have been introduced. Pardoux and Zhang [21] introduced a new class of BSDEs, which involves the integral with respect to a continuous increasing process. This kind of equations is called generalized BSDEs.

In $[20,21]$, the main ingredient is the classical martingale representation theorem. In [16], Nualart and Schoutens proved a martingale representation theorem for Lévy processes, then in [17] they established the existence and uniqueness of solution for BSDEs associated with Lévy process. Bahlali et al. [1] showed the same result for the BSDEs driven by a Brownian motion and the martingales of Teugels associated with an independent Lévy process, having a Lipschitz or a locally Lipschitz coefficient.

The aim of this paper is to study the one-dimensional generalized BSDE driven by a Brownian motion and the martingales of Teugels associated to a pure jump-independent Lévy process. We prove existence and uniqueness of the solution when the coefficient verifies some conditions of Lipschitz. In this setting, we deal with both constant and random terminal times. If the coefficient is left continuous, increasing, and bounded, we prove the existence of a solution. As an application, we give a probabilistic interpretation for large class of partial differential integral equations (PDIEs) with Neumann (nonlinear) boundary condition.

The rest of the paper is organized as follows. In Section 2, we introduce some notations. In Section 3, we prove the existence and uniqueness of the solution of the generalized BSDE when the coefficient is monotone in $y$ and uniformly Lipschitz in $z$ and $u$. Section 4 is devoted to study the case where the coefficient is left continuous in $y$, increasing, and bounded. Finally, we give in Section 5 a probabilistic interpretation of PDIE with Neumann boundary condition, and we introduce some examples of PDIE.

\section{Preliminaries}

Let $\left(\Omega, \mathscr{F},\left(\mathscr{F}_{t}\right)_{t \in[0, T]}, \mathbb{P}\right)$ be a complete probability space. $\left(\mathscr{F}_{t}\right)_{t \in[0, T]}$ is a right-continuous filtration $\left(\mathscr{F}_{t}=\bigcap_{\epsilon>0} \mathscr{F}_{t+\epsilon}=\mathscr{F}_{t^{+}}\right)$generated by $\left(W_{t}\right)_{t \in[0, T]}$, a standard Brownian motion in $\mathbb{R}$, and a Lévy process $L_{t}=b t+l_{t}$, where $l_{t}$ is a pure jumps process, corresponding to a standard Lévy measure $v$ defined in $\mathbb{R} \backslash\{0\}$ satisfying

(i) $\int_{\mathbb{R}}\left(1 \wedge x^{2}\right) \nu(d x)<+\infty$,

(ii) for some $\lambda>0$ and every $\epsilon>0, \int_{(-\epsilon, \epsilon)^{c}} e^{\lambda|x|} \nu(d x)<+\infty$.

$\left(\mathscr{F}_{t}\right)_{t \in[0, T]}$ is completed by $\mathcal{N}$, the totality of $\mathbb{P}$-null sets.

For every $\lambda \in \mathbb{R}, \mu \geq 0$, every increasing process $\left(A_{t}\right)_{t}$ and every Hilbert space $H$, we denote

(i) $\ell^{2}=\left\{x=\left(x_{n}\right)_{n \geq 0} \in \mathbb{R}^{\mathbb{N}} /\|x\|^{2}=\sum_{n=0}^{+\infty}\left|x_{n}\right|^{2}<+\infty\right\}$,

(ii) $\mathcal{H}_{\lambda, \mu}^{2}(A, H)$ is the set formed by $H$-valued progressively measurable processes $\left(X_{t}\right)_{t \geq 0}$ such that

$$
\mathbb{E}\left(\int_{0}^{T} e^{\lambda s+\mu A_{s}}\left\|X_{s}\right\|_{H}^{2} d s+\int_{0}^{T} e^{\lambda s+\mu A_{s}}\left\|X_{s}\right\|_{H}^{2} d A_{s}\right)<\infty
$$


and $\mathcal{H}_{\lambda, \mu}^{2}(H)$ is the same space satisfying

$$
\mathbb{E} \int_{0}^{T} e^{\lambda s+\mu A_{s}}\left\|X_{s}\right\|_{H}^{2} d s<\infty
$$

(iii) $\mathscr{Y}_{\lambda, \mu}^{2}(\mathbb{R})$ is the subspace of $\mathscr{H}_{\lambda, \mu}^{2}(\mathbb{R})$ of the processes $\left(Y_{t}\right)_{t \geq 0}$ satisfying

$$
\mathbb{E} \sup _{0 \leq t \leq T} e^{\lambda t+\mu A_{t}}\left|Y_{t}\right|^{2}<\infty
$$

(iv) $\mathscr{H}_{\lambda, \mu}^{2}=\mathscr{H}_{\lambda, \mu}^{2}(A, \mathbb{R}) \times \mathscr{H}_{\lambda, \mu}^{2}(\mathbb{R}) \times \mathscr{H}_{\lambda, \mu}^{2}\left(\ell^{2}\right)$ and $\mathscr{H}^{2}=\mathscr{H}_{0,0}^{2}$,

(v) $\mathscr{H}_{\lambda}^{2}=\mathscr{H}_{\lambda, 0}^{2}(\mathbb{R}) \times \mathscr{H}_{\lambda, 0}^{2}(\mathbb{R}) \times \mathscr{H}_{\lambda, 0}^{2}\left(\ell^{2}\right)$,

(vi) $\mathscr{H}_{\lambda}^{2}\left(\mathbb{R}, \ell^{2}\right)=\mathscr{H}_{\lambda, 0}^{2}(\mathbb{R}) \times \mathscr{H}_{\lambda, 0}^{2}\left(\ell^{2}\right)$.

We put $L_{t^{-}}=\lim _{s>t} L_{s}$ and $\Delta L_{t}=L_{t}-L_{t^{-}}$. We define the so-called power-jump processes $L_{t}^{(1)}=L_{t}$ and $L_{t}^{(i)}=\sum_{0<s \leq t}\left(\Delta L_{s}\right)^{i}, i \geq 2$.

Let $m_{1}=\mathbb{E} L_{1}$ and $m_{i}=\int_{-\infty}^{+\infty} x^{i} v(d x), i \geq 2$.

For all $i \geq 1$, we put $Y_{t}^{(i)}=L_{t}^{(i)}-m_{i} t$, called Teugels martingales.

We associated with the Lévy process $\left(L_{t}\right)_{t}$ the family of processes $\left(H^{(i)}\right)_{i \geq 1}$ defined by $H_{t}^{(i)}=\sum_{j=1}^{i} a_{i j} Y_{t}^{(j)}$. The coefficients $a_{i j}$ correspond to the orthonormalization of the polynomials $1, X, X^{2}, \ldots$ with respect to the measure $\pi$ defined by $\pi(d x)=x^{2} \nu(d x)$. We set for $i \geq 1$,

$$
p_{i}(x)=a_{i, i} x^{i}+a_{i, i-1} x^{i-1}+\cdots+a_{i, 1} x
$$

The martingales $H^{(i)}$ are strongly orthogonal (i.e., $H^{(i)} H^{(j)}$ is a martingale $\Leftrightarrow\left[H^{(i)}, H^{(j)}\right]$ is a martingale) and $\left\langle H^{(i)}, H^{(j)}\right\rangle_{t}=\delta_{i j} q_{i} t$, where $q_{i}=\sum_{j, k=1}^{i} a_{i j} a_{i k} m_{j+k}$ (for more details, see $[16])$.

Let us give the data $(\xi, f, g, A)$ defined by

(i) a terminal value $\xi \in \mathbb{L}^{2}\left(\Omega, \mathbb{F}_{T}, \mathbb{P}\right)$,

(ii) a map $f: \Omega \times \mathbb{R} \times \mathbb{R} \times \mathbb{R} \times \ell^{2} \rightarrow \mathbb{R}$, and $g: \Omega \times \mathbb{R} \times \mathbb{R} \rightarrow \mathbb{R}$,

(iii) a continuous one-dimensional increasing $\mathscr{F}_{t}$-progressively measurable process $\left(A_{t}\right)_{t \in[0, T]}$ satisfying $A_{0}=0$.

In the following, $C$ denotes a generic constant, that may take different values from line to line.

\section{Generalized BSDEs driven by a Lévy process on a finite interval}

In this section, we propose to show the existence and uniqueness of the solution of generalized BSDE driven by a Brownian motion and independent Lévy process (GBSDEL).

Given the data $(\xi, f, g, A)$, we introduce for all $t \in[0, T]$ the GBSDEL:

$$
Y_{t}=\xi+\int_{t}^{T} f\left(s, Y_{s^{-}}, Z_{s}, U_{s}\right) d s+\int_{t}^{T} g\left(s, Y_{s^{-}}\right) d A_{s}-\int_{t}^{T} Z_{s} d W_{s}-\sum_{i=1}^{+\infty} \int_{t}^{T} U_{s}^{(i)} d H_{s}^{(i)}
$$


We assume that for some constant $\alpha \in \mathbb{R}, \beta<0, \mu \geq 0$, and $K>0$, some adapted processes $\left\{\varphi_{t}, \psi_{t} ; 0 \leq t \leq T\right\}$ with values in $[1,+\infty)$ for all $t \in[0, T]$ and $(y, z, u),\left(y^{\prime}, z^{\prime}, u^{\prime}\right) \in \mathbb{R} \times$ $\mathbb{R} \times \ell^{2}$ are

(3.i) $\mathbb{E}\left(e^{\mu A_{T}}|\xi|^{2}\right)<+\infty$,

(3.ii) $f(\cdot, y, z, u)$ and $g(\cdot, y)$ are progressively measurable,

(3.iii) $\left(y-y^{\prime}\right)\left(f(t, y, z, u)-f\left(t, y^{\prime}, z, u\right)\right) \leq \alpha\left|y-y^{\prime}\right|^{2}$,

(3.iv) $f$ is uniformly $K$-Lipschitz with respect to $(z, v)$, that is,

$$
\mathbb{P} \text {-a.s }\left|f(t, y, z, v)-f\left(t, y, z^{\prime}, v^{\prime}\right)\right| \leq K\left(\left|z-z^{\prime}\right|+\left\|v-v^{\prime}\right\|\right),
$$

(3.v) $\left(y-y^{\prime}\right)\left(g(t, y)-g\left(t, y^{\prime}\right)\right) \leq \beta\left|y-y^{\prime}\right|^{2}$,

(3.vi) $|f(t, y, z, u)| \leq \varphi_{t}+K(|y|+|z|+\|u\|)$ and $|g(t, y)| \leq \psi_{t}+K|y|$,

(3.vii) $\mathbb{E}\left(\int_{0}^{T} e^{\mu A_{t}}\left|\varphi_{t}\right|^{2} d t+\int_{0}^{T} e^{\mu A_{t}}\left|\psi_{t}\right|^{2} d A_{t}\right)<+\infty$,

(3.viii) $y \mapsto(f(t, y, z, u), g(t, y))$ is continuous for all $(t, z, u)$ a.s.

Definition 3.1. A solution of GBSDEL is a triplet $(Y, Z, U)$ of progressively measurable processes satisfying (3.1) such that

$$
\mathbb{E}\left(\sup _{0 \leq t \leq T}\left|Y_{t}\right|^{2}+\int_{0}^{T}\left(\left|Z_{t}\right|^{2}+\left\|U_{t}\right\|^{2}\right) d t+\int_{0}^{T}\left|Y_{t}\right|^{2} d A_{t}\right)<+\infty .
$$

The objective of this section is to prove the next results.

THeOrem 3.2. Under the assumptions (3.i)-(3.viii), the GBSDEL (3.1) has a unique solution.

We want next to state an analogous result in the case where the terminal time is replaced by a stopping time $\tau$. More precisely, we consider the BSDE:

$$
\begin{aligned}
& Y_{t}= Y_{T}+\int_{t \wedge \tau}^{T \wedge \tau} f\left(s, Y_{s^{-}}, Z_{s}, U_{s}\right) d s+\int_{t \wedge \tau}^{T \wedge \tau} g\left(s, Y_{s^{-}}\right) d A_{s}-\int_{t \wedge \tau}^{T \wedge \tau} Z_{s} d W_{s} \\
&-\sum_{i=1}^{+\infty} \int_{t \wedge \tau}^{T \wedge \tau} U_{s}^{(i)} d H_{s}^{(i)}, \quad \forall 0 \leq t \leq T<\infty, \\
& Y_{t}=\xi \quad \text { on the set }\{t \geq \tau\} .
\end{aligned}
$$

We assume that $\xi$ is an $\mathscr{F}_{\tau}$-measurable, and that for some $\lambda>2 \alpha+4 K^{2}, \mu>2 \beta$.

(3.ii)'

$$
\mathbb{E}\left(\int_{0}^{\infty} e^{\lambda t+\mu A_{t}}\left(\left|\varphi_{t}\right|^{2}+\left|f\left(t, \xi_{t}, \zeta_{t}, \rho_{t}\right)\right|^{2}\right) d t+\int_{0}^{\infty} e^{\lambda t+\mu A_{t}}\left(\left|\psi_{t}\right|^{2}+\left|g\left(t, \xi_{t}\right)\right|^{2}\right) d A_{t}\right)<\infty,
$$

where $\xi_{t}=\mathbb{E}\left(\xi / \mathscr{F}_{t}\right), \zeta$ and $\rho=\left(\rho^{(i)}\right)_{i=1}^{\infty}$ are progressively measurable processes such that $\mathbb{E} \int_{0}^{\tau}\left(\left|\zeta_{t}\right|^{2}+\left\|\rho_{t}\right\|^{2}\right) d t<\infty$, and

$$
\xi=\mathbb{E}(\xi)+\int_{0}^{\tau} \zeta_{t} d W_{t}+\sum_{i=1}^{+\infty} \int_{0}^{\tau} \rho_{t}^{(i)} d H_{t}^{(i)} .
$$


The existence of $\left(\zeta_{t}\right)_{t}$ and $\left(\rho_{t}\right)_{t}$ is insured by combining the results of Løkka [14] and Nualart and Schoutens [16].

(3.viii) $\mathbb{E}\left(\left(1+e^{\lambda \tau+\mu A_{\tau}}\right)|\xi|^{2}\right)<\infty$.

The result which we want to prove is the following.

Theorem 3.3. Under the conditions (3.i), (3.ii)', (3.iii)-(3.vii), (3.viii)', there exists a unique progressively measurable process $\left(Y_{t}, Z_{t}, U_{t}\right)_{0 \leq t \leq \tau}$ solution of (3.4), such that for some $\lambda>2 \alpha+4 K^{2}$ and $\mu>2 \beta$,

$$
\mathbb{E}\left(\sup _{0 \leq t \leq \tau}\left|Y_{t}\right|^{2}+\int_{0}^{\tau} e^{\lambda t+\mu A_{t}}\left|Y_{t}\right|^{2} d A_{t}+\int_{0}^{\tau} e^{\lambda t+\mu A_{t}}\left(\left|Z_{t}\right|^{2}+\|\left. U_{t}\right|^{2}\right) d t\right)<+\infty .
$$

Remark 3.4. By Itô's formula, we can write

$$
\begin{aligned}
e^{\mu A_{t}} Y_{t}= & e^{\mu A_{T}} \xi+\int_{t}^{T} e^{\mu A_{s}} f\left(s, Y_{s^{-}}, Z_{s}, U_{s}\right) d s+\int_{t}^{T} e^{\mu A_{s}}\left(g\left(s, Y_{s^{-}}\right)-\mu Y_{s^{-}}\right) d A_{s} \\
& -\int_{t}^{T} e^{\mu A_{s}} Z_{s} d W_{s}-\sum_{i=1}^{+\infty} \int_{t}^{T} e^{\mu A_{s}} U_{s}^{(i)} d H_{s}^{(i)}+\mu \sum_{i=1}^{+\infty}\left[\int_{0}^{\cdot} e^{\mu A_{s}} d A_{s}, \int_{0}^{\cdot} U_{s}^{(i)} d H_{s}^{(i)}\right]_{t}^{T},
\end{aligned}
$$

for all $i \geq 1$, the process $\left(\int_{0}^{t} U_{s}^{(i)} d H_{s}^{(i)}\right)_{t \geq 0}$ is a pure jumps process, then by [23, Theorem 26 , page 75], the last term is equal to 0 . So, if $\left(Y_{t}, Z_{t}, U_{t}\right)$ satisfies (3.1), then

$$
\left(\bar{Y}_{t}, \bar{Z}_{t}, \bar{U}_{t}\right)=\left(e^{\mu A_{t}} Y_{t}, e^{\mu A_{t}} Z_{t}, e^{\mu A_{t}} U_{t}\right)
$$

satisfies an analogous GBSDEL with $f$ and $g$ replaced by

$$
\begin{gathered}
\bar{f}(t, y, z, u)=e^{\mu A_{t}} f\left(t, e^{-\mu A_{t}} y, e^{-\mu A_{t}} z, e^{-\mu A_{t}} u\right), \\
\bar{g}(t, y)=e^{\mu A_{t}} g\left(t, e^{-\mu A_{t}} y\right)-\mu y .
\end{gathered}
$$

Hence, if $g$ satisfies (3.v) with a possibly nonnegative $\beta$, we can always choose $\mu$ such that $\bar{g}$ satisfies (3.v) with a strictly negative $\bar{\beta}$.

3.1. Preliminary estimates and uniqueness. We first establish a priori estimate on the solution.

Proposition 3.5. Under the conditions (3.i)-(3.viii), if $(Y, Z, U)$ is solution of (3.1), then there exists a constant $C>0$ only depending on $\alpha, \beta, K$, and $T$, such that

$$
\begin{aligned}
& \mathbb{E}\left(\sup _{0 \leq t \leq T}\left|Y_{t}\right|^{2}+\int_{0}^{T}\left|Y_{t}\right|^{2} d A_{t}+\int_{0}^{T}\left(\left|Z_{t}\right|^{2}+\|\left. U_{t}\right|^{2}\right) d t\right) \\
& \leq C\left(\mathbb{E}|\xi|^{2}+\mathbb{E} \int_{0}^{T}|f(t, 0,0,0)|^{2} d t+\mathbb{E} \int_{0}^{T}|g(t, 0)|^{2} d A_{t}\right) .
\end{aligned}
$$


6 Generalized BSDE driven by a Lévy process

Proof. From Itô's formula,

$$
\begin{aligned}
|\xi|^{2}= & \left|Y_{t}\right|^{2}-2 \int_{t}^{T} Y_{s^{-}} f\left(s, Y_{s^{-}}, Z_{s}, U_{s}\right) d s-2 \int_{t}^{T} Y_{s^{-}} g\left(s, Y_{s^{-}}\right) d A_{s}+\int_{t}^{T}\left|Z_{s}\right|^{2} d s \\
& +2 \int_{t}^{T} Y_{s} Z_{s} d W_{s}+2 \sum_{i=1}^{\infty} \int_{t}^{T} Y_{s} U_{s}^{(i)} d H_{s}^{(i)}+\sum_{i, j=1}^{\infty} \int_{t}^{T} U_{s}^{(i)} U_{s}^{(j)} d\left[H^{(i)}, H^{(j)}\right]_{s^{.}}
\end{aligned}
$$

Let us note that $\left(\int_{0}^{t} Y_{s} Z_{s} d W_{s}\right)_{0 \leq t \leq T},\left(\int_{0}^{t} Y_{s} U_{s}^{(i)} d H_{s}^{(i)}\right)_{0 \leq t \leq T}$ for all $i \geq 1$ and $\left(\int_{0}^{t} U_{s}^{(i)} U_{s}^{(j)} d\left[H^{(i)}\right.\right.$, $\left.\left.H^{(j)}\right]_{s}\right)_{0 \leq t \leq T}$ for $i \neq j$ are uniformly integrable martingales.

Taking the expectation, we get

$$
\begin{aligned}
\mathbb{E}\left|Y_{t}\right|^{2} & +\mathbb{E} \int_{t}^{T}\left(\left|Z_{s}\right|^{2}+\left\|U_{s}\right\|^{2}\right) d s \\
\quad & =\mathbb{E}|\xi|^{2}+2 \mathbb{E} \int_{t}^{T} Y_{s} f\left(s, Y_{s}, Z_{s}, U_{s}\right) d s+2 \mathbb{E} \int_{t}^{T} Y_{s} g\left(s, Y_{s}\right) d A_{s} .
\end{aligned}
$$

On the other hand, by (3.iii)-(3.v), we can write

$$
\begin{aligned}
Y_{s} f\left(s, Y_{s}, Z_{s}, U_{s}\right) & \leq \alpha\left|Y_{s}\right|^{2}+\left|Y_{s}\right|\left(|f(s, 0,0,0)|+K\left(\left|Z_{s}\right|+|| U_{s}||\right)\right) \\
& \leq(2 \alpha+1)\left|Y_{s}\right|^{2}+|f(s, 0,0,0)|^{2}+\frac{2 K^{2}}{\alpha}\left(\left|Z_{s}\right|^{2}+|| U_{s} \|^{2}\right) \\
Y_{s} g\left(s, Y_{s}\right) & \leq \beta\left|Y_{s}\right|^{2}+\left|Y_{s}\right||g(s, 0)| \\
& \leq-\frac{|\beta|}{2}\left|Y_{s}\right|^{2}+\frac{2}{|\beta|}|g(s, 0)|^{2}
\end{aligned}
$$

Consequently, for $\alpha \geq 2 K^{2}$, we can write

$$
\begin{aligned}
\mathbb{E}\left|Y_{t}\right|^{2} & +\mathbb{E} \int_{t}^{T}\left|Y_{s}\right|^{2} d A_{s}+\mathbb{E} \int_{t}^{T}\left(\left|Z_{s}\right|^{2}+\| U_{s}||^{2}\right) d s \\
& \leq C \mathbb{E}\left(|\xi|^{2}+\int_{0}^{T}|f(s, 0,0,0)|^{2} d s+\int_{0}^{T}|g(s, 0)|^{2} d A_{s}+\int_{t}^{T}\left|Y_{s}\right|^{2} d s\right) .
\end{aligned}
$$

By the Gronwall lemma, we conclude that

$$
\begin{array}{rl}
\sup _{0 \leq t \leq T} & \mathbb{E}\left|Y_{t}\right|^{2}+\mathbb{E} \int_{0}^{T}\left(\left|Z_{s}\right|^{2}+\left.|| U_{s}\right|^{2}\right) d s+\mathbb{E} \int_{0}^{T}\left|Y_{s}\right|^{2} d A_{s} \\
& \leq C\left(\mathbb{E}|\xi|^{2}+\mathbb{E} \int_{0}^{T}|f(s, 0,0,0)|^{2} d s+\mathbb{E} \int_{0}^{T}|g(s, 0)|^{2} d A_{s}\right) .
\end{array}
$$

The result follows from this and from Burkhölder-Davis-Gundy inequality.

Proposition 3.6. Under the assumption (3.i)-(3.viii), there exists at most one progressively measurable process $\left(Y_{t}, Z_{t}, U_{t}\right)_{0 \leq t \leq T}$ solution of (3.1). 
Proof. Let $\left(Y^{1}, Z^{1}, U^{1}\right)$ and $\left(Y^{2}, Z^{2}, U^{2}\right)$ be two solutions of (3.1).

We denote by

$$
\begin{gathered}
\delta Y_{s}=Y_{s}^{1}-Y_{s}^{2}, \quad \delta Z_{s}=Z_{s}^{1}-Z_{s}^{2}, \quad \delta U_{s}=U_{s}^{1}-U_{s}^{2}, \\
\delta f_{s}=f\left(s, Y_{s}^{1}, Z_{s}^{1}, U_{s}^{1}\right)-f\left(s, Y_{s}^{2}, Z_{s}^{2}, U_{s}^{2}\right), \quad \delta g_{s}=g\left(s, Y_{s}^{1}\right)-g\left(s, Y_{s}^{2}\right) .
\end{gathered}
$$

From Itô's formula,

$$
\mathbb{E}\left|\delta Y_{t}\right|^{2}+\mathbb{E} \int_{t}^{T}\left(\left|\delta Z_{s}\right|^{2}+\left\|\delta U_{s}\right\|^{2}\right) d s=2 \mathbb{E} \int_{t}^{T} \delta Y_{s} \delta f_{s} d s+2 \mathbb{E} \int_{t}^{T} \delta Y_{s} \delta g_{s} d A_{s} .
$$

However, by (3.iii)-(3.v), we have

$$
\begin{gathered}
\delta Y_{s} \delta f_{s} \leq 2 \alpha\left|\delta Y_{s}\right|^{2}+\frac{2 K^{2}}{\alpha}\left(\left|\delta Z_{s}\right|^{2}+\left\|\delta U_{s}\right\|^{2}\right) \\
\delta Y_{s} \delta g_{s} \leq \beta\left|\delta Y_{s}\right|^{2} .
\end{gathered}
$$

Substituting these inequalities, we obtain

$$
\begin{aligned}
& \mathbb{E}\left|\delta Y_{s}\right|^{2}+2|\beta| \mathbb{E} \int_{t}^{T}\left|\delta Y_{s}\right|^{2} d A_{s}+\left(1-\frac{4 K^{2}}{\alpha}\right) \mathbb{E} \int_{t}^{T}\left(\left|\delta Z_{s}\right|^{2}+\left\|\delta U_{s}\right\|^{2}\right) d s \\
& \leq 4 \alpha \mathbb{E} \int_{t}^{T}\left|\delta Y_{s}\right|^{2} d s .
\end{aligned}
$$

For $\alpha \geq 4 K^{2}$, we conclude by the Gronwall lemma that $(\delta Y, \delta Z, \delta U)=(0,0,0)$.

3.2. Existence result of GBSDEL on fixed finite time interval. We first prove existence and uniqueness result under an additional assumption.

We suppose that for all $y, y^{\prime}, z \in \mathbb{R}$ and $u \in \ell^{2}, d t \times d \mathbb{P}$ a.e.

$$
\left|f(t, y, z, u)-f\left(t, y^{\prime}, z, u\right)\right|+\left|g(t, y)-g\left(t, y^{\prime}\right)\right| \leq K\left|y-y^{\prime}\right|
$$

Theorem 3.7. Under the assumptions (3.i), (3.ii), (3.iv)-(3.ix), there exists a unique progressively measurable process $\left(Y_{t}, Z_{t}, U_{t}\right)_{0 \leq t \leq T}$ solution of (3.1).

Proof. First let us assume that the map $f$ does not depend on $(y, z, v)$. Using the martingale representation theorem, we can prove that the following GBSDE:

$$
Y_{t}=\xi+\int_{t}^{T} f(s) d s+\int_{t}^{T} g(s) d A_{s}-\int_{t}^{T} Z_{s} d W_{s}-\sum_{i=1}^{+\infty} \int_{t}^{T} U_{s}^{(i)} d H_{s}^{(i)}
$$

has a unique solution that verifies (3.3).

Now, define the sequence $\left(Y^{n}, Z^{n}, U^{n}\right)$ as follows. 
$\left(Y^{0}, Z^{0}, U^{0}\right)=(0,0,0)$, and $\left(Y^{n+1}, Z^{n+1}, U^{n+1}\right)$ is the unique solution of the BSDE:

$$
\begin{aligned}
Y_{t}^{n+1}= & \xi+\int_{t}^{T} f\left(s, Y_{s^{-}}^{n}, Z_{s}^{n}, U_{s}^{n}\right) d s+\int_{t}^{T} g\left(s, Y_{s^{-}}^{n}\right) d A_{s}-\int_{t}^{T} Z_{s}^{n+1} d W_{s} \\
& -\sum_{i=1}^{\infty} \int_{t}^{T} U_{s}^{n+1(i)} d H_{s}^{(i)} .
\end{aligned}
$$

We will prove that $\left(Y^{n}, Z^{n}, U^{n}\right)$ is a Cauchy sequence in the Banach space $\mathcal{H}_{\lambda, \mu}^{2}$.

Note that for some $(\lambda, \mu)$, we can show that

$$
\begin{gathered}
\sup _{n \geq 0}\left(\mathbb{E} \sup _{0 \leq t \leq T} e^{\lambda t+\mu A_{t}}\left|Y_{t}^{n}\right|^{2}+\mathbb{E} \int_{0}^{T} e^{\lambda t+\mu A_{t}}\left|Y_{t}^{n}\right|^{2} d t+\mathbb{E} \int_{0}^{T} e^{\lambda t+\mu A_{t}}\left|Y_{t}^{n}\right|^{2} d A_{t}\right. \\
\left.+\mathbb{E} \int_{0}^{T} e^{\lambda t+\mu A_{t}}\left(\left|Z_{t}^{n}\right|^{2}+\left\|U_{t}^{n}\right\|^{2}\right) d t\right) \leq C .
\end{gathered}
$$

To simplify, we put for $n \geq m \geq 1$ and $0 \leq s \leq T$

$$
\begin{gathered}
\bar{Y}_{s}^{n, m}=Y_{s}^{n}-Y_{s}^{m}, \quad \bar{Z}_{s}^{n, m}=Z_{s}^{n}-Z_{s}^{m}, \quad \bar{U}_{s}^{n, m}=U_{s}^{n}-U_{s}^{m}, \\
\bar{f}_{s}^{n, m}=f\left(s, Y_{s}^{n}, Z_{s}^{n}, U_{s}^{n}\right)-f\left(s, Y_{s}^{m}, Z_{s}^{m}, U_{s}^{m}\right), \quad \bar{g}_{s}^{n, m}=g\left(s, Y_{s}^{n}\right)-g\left(s, Y_{s}^{m}\right) .
\end{gathered}
$$

If we apply Itô's formula and if we take the expectation, we have

$$
\begin{aligned}
\mathbb{E} e^{\lambda t+\mu A_{t}}\left|\bar{Y}_{t}^{n+1, m+1}\right|^{2}+\lambda \mathbb{E} \int_{t}^{T} e^{\lambda s+\mu A_{s}}\left|\bar{Y}_{s}^{n+1, m+1}\right|^{2} d s+\mu \mathbb{E} \int_{t}^{T} e^{\lambda s+\mu A_{s}}\left|\bar{Y}_{s}^{n+1, m+1}\right|^{2} d A_{s} \\
\quad+\mathbb{E} \int_{t}^{T} e^{\lambda s+\mu A_{s}}\left(\left|\bar{Z}_{s}^{n+1, m+1}\right|^{2}+\left.|| \bar{U}_{s}^{n+1, m+1}\right|^{2}\right) d s \\
=2 \mathbb{E} \int_{t}^{T} e^{\lambda s+\mu A_{s}} \bar{Y}_{s}^{n+1, m+1} \bar{f}_{s}^{n, m} d s+2 \mathbb{E} \int_{t}^{T} e^{\lambda s+\mu A_{s}} \bar{Y}_{s}^{n+1, m+1} \bar{g}_{s}^{n, m} d A_{s} \\
\leq 6 K^{2} \mathbb{E} \int_{t}^{T} e^{\lambda s+\mu A_{s}}\left|\bar{Y}_{s}^{n+1, m+1}\right|^{2} d s+2 K^{2} \mathbb{E} \int_{t}^{T} e^{\lambda s+\mu A_{s}}\left|\bar{Y}_{s}^{n+1, m+1}\right|^{2} d A_{s} \\
\quad+\frac{1}{2} \mathbb{E} \int_{t}^{T} e^{\lambda s+\mu A_{s}}\left(\left|\bar{Y}_{s}^{n, m}\right|^{2}+\left|\bar{Z}_{s}^{n, m}\right|^{2}+|| \bar{U}_{s}^{n, m}||^{2}\right) d s+\frac{1}{2} \mathbb{E} \int_{t}^{T} e^{\lambda s+\mu A_{s}}\left|\bar{Y}_{s}^{n, m}\right|^{2} d A_{s} .
\end{aligned}
$$

Choosing $\lambda=1+6 K^{2}$ and $\mu=1+2 K^{2}$, we deduce that

$$
\begin{aligned}
\left\|\left(\bar{Y}^{n+1, m+1}, \bar{Z}^{n+1, m+1}, \bar{U}^{n+1, m+1}\right)\right\|_{\mathscr{H} \ell_{\lambda, \mu}^{2}}^{2} & \leq \frac{1}{2}\left\|\left(\bar{Y}^{n, m}, \bar{Z}^{n, m}, \bar{U}^{n, m}\right)\right\|_{\mathscr{H}_{\lambda, \mu}^{2}}^{2} \\
& \leq\left(\frac{1}{2}\right)^{m+1}\left\|\left(Y^{n-m}, Z^{n-m}, U^{n-m}\right)\right\|_{\mathscr{H} \mathscr{H}_{\lambda, \mu}^{2}}^{2} \\
& \leq \frac{C}{2^{m+1}} \stackrel{m \rightarrow \infty}{\longrightarrow} 0 .
\end{aligned}
$$


Consequently, the sequence $\left(Y^{n}, Z^{n}, U^{n}\right)$ converges in the Banach space $\mathcal{H}_{\lambda, \mu}^{2}$ to a process $(Y, Z, U)$, that is not difficult to show that verifies (3.1).

We now establish existence and uniqueness for (3.1) under the conditions (3.i)-(3.viii). First, we need the following proposition.

Proposition 3.8. Given $(\tilde{Z}, \tilde{U}) \in \mathscr{H}_{0}^{2}\left(\mathbb{R}, \ell^{2}\right)$, there exists a unique progressively measurable process $\left(Y_{t}, Z_{t}, U_{t}\right)_{0 \leq t \leq T}$ solution of

$$
Y_{t}=\xi+\int_{t}^{T} f\left(s, Y_{s^{-}}, \tilde{Z}_{s}, \tilde{U}_{s}\right) d s+\int_{t}^{T} g\left(s, Y_{s^{-}}\right) d A_{s}-\int_{t}^{T} Z_{s} d W_{s}-\sum_{i=1}^{+\infty} \int_{t}^{T} U_{s}^{(i)} d H_{s}^{(i)} .
$$

Proof. The proof is very similar to that of [21, Proposition 1.8].

To simplify, we put $\tilde{f}(s, y)=f\left(s, y, \widetilde{Z}_{s}, \widetilde{U}_{s}\right)$. Notice that $f(s, y)$ satisfies the following.

(3.iii) $)^{\prime}\left(y-y^{\prime}\right)\left(\tilde{f}(s, y)-\tilde{f}\left(s, y^{\prime}\right)\right) \leq \alpha\left|y-y^{\prime}\right|^{2}$.

(3.vi) $)^{\prime}|\tilde{f}(t, y)| \leq \tilde{\varphi}_{t}+K|y|$.

(3.vii) $)^{\prime} \mathbb{E} \int_{0}^{T}\left|\tilde{\varphi}_{t}\right|^{2} d t<\infty$.

(3.viii)' $y \mapsto \tilde{f}(t, y)$ is continuous $d \mathbb{P} \times d t$ a.e.

We approximate $\tilde{f}$ and $g$ by $\tilde{f}_{n}$ and $g_{n}$ such that

(i) for each $n, \tilde{f}_{n}$ and $g_{n}$ are uniformly Lipschitz in $y$,

(ii) $f_{n}$ satisfies (3.iii) ${ }^{\prime}$ and (3.vi)', and $g_{n}$ satisfies (3.v) and (3.vi) with fixed constants $\alpha, \beta, K$ and fixed process $\left\{\left(\tilde{\varphi}_{t}\right)_{t},\left(\psi_{t}\right)_{t}\right\}_{0 \leq t \leq T}$ satisfying (3.vii)' and (3.vii).

For each $n$, there exists a unique progressively measurable process $\left(\tilde{Y}^{n}, \widetilde{Z}^{n}, \tilde{U}^{n}\right)$ solution of (3.1), such that

$$
\sup _{n \geq 0} \mathbb{E}\left(\sup _{0 \leq t \leq T}\left|\tilde{Y}_{t}^{n}\right|^{2}+\int_{0}^{T}\left|\tilde{Y}_{t}^{n}\right|^{2} d A_{t}+\int_{0}^{T}\left(\left|\tilde{Z}_{t}^{n}\right|^{2}+\left\|\tilde{U}_{t}^{n}\right\|^{2}\right) d t\right)<\infty .
$$

Defining $\widetilde{V}_{t}^{n}=\widetilde{f}_{n}\left(t, \widetilde{Y}_{t}^{n}\right)$ and $\widetilde{W}_{t}^{n}=g\left(t, \widetilde{Y}_{t}^{n}\right)$, we deduce from the above and from our assumptions that

$$
\sup _{n \geq 0} \mathbb{E}\left(\int_{0}^{T}\left|\tilde{V}_{t}^{n}\right|^{2} d t+\int_{0}^{T}\left|\widetilde{W}_{t}^{n}\right|^{2} d A_{t}\right)<\infty
$$

From weak convergence along a subsequence, we conclude that there exists a progressively measurable process $\left(Y_{t}, Z_{t}, U_{t}, \widetilde{V}_{t}, \widetilde{W}_{t}\right)_{0 \leq t \leq T}$ verifying

$$
Y_{t}=\xi+\int_{t}^{T} \tilde{V}_{s} d s+\int_{t}^{T} \widetilde{W}_{s} d A_{s}-\int_{t}^{T} Z_{s} d W_{s}-\sum_{i=1}^{+\infty} \int_{t}^{T} U_{s}^{(i)} d H_{s}^{(i)}
$$

Finally, we can show that $\widetilde{V}_{t}=\tilde{f}\left(t, Y_{t}\right)$ and $\widetilde{W}_{t}=g\left(t, Y_{t}\right)$. 
Let $X$ and $X^{\prime}$ be two progressively measurable processes such that

$$
\begin{gathered}
\mathbb{E} \int_{0}^{T}\left|X_{t}\right|^{2} d t<\infty, \quad \mathbb{E} \int_{0}^{T}\left|X_{t}^{\prime}\right|^{2} d A_{t}<\infty, \\
\mathbb{E} \int_{0}^{T} e^{\alpha t}\left(\tilde{Y}_{t}^{n}-X_{t}\right)\left(\tilde{f}_{n}\left(t, \tilde{Y}_{t}^{n}\right)-\tilde{f}_{n}\left(t, X_{t}\right)-\alpha\left(\tilde{Y}_{t}^{n}-X_{t}\right)\right) d t \\
+\mathbb{E} \int_{0}^{T} e^{\alpha t}\left(\tilde{Y}_{t}^{n}-X_{t}^{\prime}\right)\left(g_{n}\left(t, \tilde{Y}_{t}^{n}\right)-g_{n}\left(t, X_{t}^{\prime}\right)\right) d A_{t} \leq 0 .
\end{gathered}
$$

Since $\mathbb{E} \int_{0}^{T}\left|\tilde{f}_{n}\left(t, X_{t}\right)-\tilde{f}\left(t, X_{t}\right)\right|^{2} d t+\mathbb{E} \int_{0}^{T}\left|g_{n}\left(t, X_{t}^{\prime}\right)-g\left(t, X_{t}^{\prime}\right)\right|^{2} d A_{t} \rightarrow 0$ as $n \rightarrow+\infty$, we can write

$$
\begin{aligned}
\lim _{n \rightarrow \infty} \sup & \mathbb{E} \int_{0}^{T} e^{\alpha t}\left(\tilde{Y}_{t}^{n}-X_{t}\right)\left(\tilde{f}_{n}\left(t, \tilde{Y}_{t}^{n}\right)-\tilde{f}\left(t, X_{t}\right)-\alpha\left(\tilde{Y}_{t}-X_{t}\right)\right) d t \\
+ & \mathbb{E} \int_{0}^{T} e^{\alpha t}\left(\tilde{Y}_{t}^{n}-X_{t}^{\prime}\right)\left(g_{n}\left(t, \tilde{Y}_{t}^{n}\right)-g\left(t, X_{t}^{\prime}\right)\right) d A_{t} \leq 0 .
\end{aligned}
$$

On the other hand, if we apply Itô's formula, we obtain

$$
\begin{gathered}
\mathbb{E} \int_{0}^{T} 2 e^{\alpha t} \tilde{Y}_{t}^{n}\left(\tilde{f}_{n}\left(t, \tilde{Y}_{t}^{n}\right)-\alpha \tilde{Y}_{t}^{n}\right) d t+2 \mathbb{E} \int_{0}^{T} e^{\alpha t} \tilde{Y}_{t}^{n} g_{n}\left(t, \tilde{Y}^{n}\right) d A_{t} \\
=\left|\tilde{Y}_{0}^{n}\right|^{2}-e^{\alpha T} \mathbb{E}|\xi|^{2}+\mathbb{E} \int_{0}^{T} e^{\alpha t}\left(\left|\tilde{Z}_{t}^{n}\right|^{2}+\left\|\tilde{U}_{t}^{n}\right\|^{2}\right) d t .
\end{gathered}
$$

Using the fact that $\tilde{Y}_{0}^{n} \rightarrow Y_{0}$ in $\mathbb{R}$, and that the mapping

$$
(Z, U) \longmapsto \mathbb{E} \int_{0}^{T} e^{\alpha t}\left(\left|Z_{t}\right|^{2}+\left\|U_{t}\right\|^{2}\right) d t
$$

is convex and continuous in $\mathscr{H}_{\alpha}^{2}\left(\mathbb{R}, \ell^{2}\right)$, we get

$$
\begin{aligned}
\lim _{n \rightarrow \infty} \inf \mathbb{E} \int_{0}^{T} 2 e^{\alpha t} \tilde{Y}_{t}^{n}\left(\tilde{f}_{n}\left(t, \tilde{Y}_{t}^{n}\right)-\alpha \tilde{Y}_{t}^{n}\right) d t+2 \mathbb{E} \int_{0}^{T} e^{\alpha t} \tilde{Y}_{t}^{n} g_{n}\left(t, \tilde{Y}_{t}^{n}\right) d A_{t} \\
\geq\left|Y_{0}\right|^{2}-e^{\alpha T} \mathbb{E}|\xi|^{2}+\mathbb{E} \int_{0}^{T} e^{\alpha t}\left(\left|Z_{t}\right|^{2}+\|\left. U_{t}\right|^{2}\right) d t \\
\quad=\mathbb{E} \int_{0}^{T} 2 e^{\alpha t} Y_{t}\left(\tilde{V}_{t}-\alpha Y_{t}\right) d t+2 \mathbb{E} \int_{0}^{T} e^{\alpha t} Y_{t} \widetilde{W}_{t} d A_{t} .
\end{aligned}
$$

Combining this inequality and (3.33), we obtain

$$
\begin{gathered}
\mathbb{E} \int_{0}^{T} e^{\alpha t}\left(Y_{t}-X_{t}\right)\left(\widetilde{V}_{t}-\tilde{f}\left(t, X_{t}\right)-\alpha\left(Y_{t}-X_{t}\right)\right) d t \\
\quad+\mathbb{E} \int_{0}^{T} e^{\alpha t}\left(Y_{t}-X_{t}^{\prime}\right)\left(\widetilde{W}_{t}-g\left(t, X_{t}^{\prime}\right)\right) d A_{t} \leq 0 .
\end{gathered}
$$


We choose $X_{t}=Y_{t}-\varepsilon\left(\tilde{V}_{t}-f\left(t, Y_{t}\right)\right), X_{t}^{\prime}=Y_{t}-\varepsilon\left(\widetilde{W}_{t}-g\left(t, Y_{t}\right)\right)$, divide them by $\varepsilon$ and let $\varepsilon \rightarrow 0$ to conclude.

Proof of Theorem 3.2. We construct a mapping $\Phi$ from $\mathscr{H}^{2}$ into itself, which to $(\tilde{Y}, \tilde{Z}, \tilde{U})$ associates $(Y, Z, U)=\Phi(\tilde{Y}, \tilde{Z}, \tilde{U})$ solution of (3.28). Our aim is to show that $\Phi$ admits a unique fixed point.

Let $(\tilde{Y}, \tilde{Z}, \widetilde{U})$ and $\left(\tilde{Y}^{\prime}, \tilde{Z}^{\prime}, \tilde{U}^{\prime}\right) \in \mathscr{H}^{2}$ such that

$$
(Y, Z, U)=\Phi(\tilde{Y}, \tilde{Z}, \tilde{U}), \quad\left(Y^{\prime}, Z^{\prime}, U^{\prime}\right)=\Phi\left(\tilde{Y}^{\prime}, \tilde{Z}^{\prime}, \tilde{U}^{\prime}\right)
$$

Denote by $\delta X_{s}=X_{s}-X_{s}^{\prime}$ for $X=Y, \tilde{Y}, Z, \tilde{Z}, U$, and $\tilde{U}$.

It follows from Itô's formula and the conditions (3.iii)-(3.v) that

$$
\begin{aligned}
e^{\gamma t} \mathbb{E} \mid & \left.\delta Y_{t}\right|^{2}+\mathbb{E} \int_{t}^{T} e^{\gamma s}\left|\delta Y_{s}\right|^{2}\left(\gamma d s+2|\beta| d A_{s}\right)+\mathbb{E} \int_{t}^{T} e^{\gamma s}\left(\left|\delta Z_{s}\right|^{2}+\left\|U_{s}\right\|^{2}\right) d s \\
\leq & 2 \alpha \mathbb{E} \int_{t}^{T} e^{\gamma s}\left|\delta Y_{s}\right|^{2} d s+2 K \mathbb{E} \int_{t}^{T} e^{\gamma s}\left|\delta Y_{s}\right|\left(\left|\delta \tilde{Z}_{s}\right|+|| \delta \tilde{U}_{s}||\right) d s \\
& \leq\left(2 \alpha+4 K^{2}\right) \mathbb{E} \int_{t}^{T} e^{\gamma s}\left|\delta Y_{s}\right|^{2} d s+\frac{1}{2} \mathbb{E} \int_{t}^{T} e^{\gamma s}\left(\left|\delta \tilde{Z}_{s}\right|^{2}+\left\|\delta \tilde{U}_{s}\right\|^{2}\right) d s .
\end{aligned}
$$

Choosing $\gamma=1+2 \alpha+4 K^{2}$, we deduce that

$$
\|(\delta Y, \delta Z, \delta U)\|_{\mathscr{H}_{\gamma}^{2}}^{2} \leq \frac{1}{2}\|(\delta \tilde{Y}, \delta \tilde{Z}, \delta \tilde{U})\|_{\mathscr{H}_{\gamma}^{2}}^{2} .
$$

It follows that $\Phi$ has a unique fixed point solution of the GBSDEL (3.1).

\subsection{Existence and uniqueness results for the GBSDEL on a random time interval}

Proof of Theorem 3.3 (uniqueness). Let $\left(Y^{1}, Z^{1}, U^{1}\right)$ and $\left(Y^{2}, Z^{2}, U^{2}\right)$ be two solutions of (3.4) and satisfy (3.7). We keep the same notations as in the proof of Proposition 3.6.

From Itô's formula and passing to the expectation,

$$
\begin{aligned}
\mathbb{E} e^{\lambda(T \wedge \tau)+\mu A_{T \wedge \tau}}\left|\delta Y_{T}\right|^{2}= & \mathbb{E} e^{\lambda(t \wedge \tau)+\mu A_{t \wedge \tau}}\left|\delta Y_{t}\right|^{2}+\mathbb{E} \int_{t \wedge \tau}^{T \wedge \tau} e^{\lambda s+\mu A_{s}}\left|\delta Y_{s}\right|^{2}\left(\lambda d s+\mu d A_{s}\right) \\
& +\mathbb{E} \int_{t \wedge \tau}^{T \wedge \tau} e^{\lambda s+\mu A_{s}}\left(\left|\delta Z_{s}\right|^{2}+\|\left.\delta U_{s}\right|^{2}\right) d s \\
& -2 \mathbb{E} \int_{t \wedge \tau}^{T \wedge \tau} e^{\lambda s+\mu A_{s}} \delta Y_{s} \delta f_{s} d s-2 \mathbb{E} \int_{t \wedge \tau}^{T \wedge \tau} e^{\lambda s+\mu A_{s}} \delta Y_{s} \delta g_{s} d A_{s} .
\end{aligned}
$$


12 Generalized BSDE driven by a Lévy process

By the assumptions (3.iii)-(3.v), we can write

$$
\begin{aligned}
& \mathbb{E} e^{\lambda(t \wedge \tau)+\mu A_{t \wedge \tau}}\left|\delta Y_{t}\right|^{2} \\
& +\lambda \mathbb{E} \int_{t \wedge \tau}^{T \wedge \tau} e^{\lambda s+\mu A_{s}}\left|\delta Y_{s}\right|^{2} d s+\mu \mathbb{E} \int_{t \wedge \tau}^{T \wedge \tau} e^{\lambda s+\mu A_{s}}\left|\delta Y_{s}\right|^{2} d A_{s} \\
& +\mathbb{E} \int_{t \wedge \tau}^{T \wedge \tau} e^{\lambda s+\mu A_{s}}\left|\delta Z_{s}\right|^{2} d s+\mathbb{E} \int_{t \wedge \tau}^{T \wedge \tau} e^{\lambda s+\mu A_{s}}|| \delta U_{s} \|^{2} d s \\
& \leq \mathbb{E} e^{\lambda(T \wedge \tau)+\mu A_{T \wedge \tau}}\left|\delta Y_{T}\right|^{2}+2 \beta \mathbb{E} \int_{t \wedge \tau}^{T \wedge \tau} e^{\lambda s+\mu A_{s}}\left|\delta Y_{s}\right|^{2} d A_{s} \\
& +2 \mathbb{E} \int_{t \wedge \tau}^{T \wedge \tau} e^{\lambda s+\mu A_{s}}\left(\alpha\left|\delta Y_{s}\right|^{2}+K\left|\delta Y_{s}\right|\left(\left|\delta Z_{s}\right|+|| \delta U_{s}||\right)\right) d s .
\end{aligned}
$$

Let $\nu=\left(\lambda-2 \alpha-4 K^{2}\right) \wedge(\mu-2 \beta)$, then

$$
\begin{aligned}
& \mathbb{E} e^{\lambda(t \wedge \tau)+\mu A_{t \wedge \tau}}\left|\delta Y_{t}\right|^{2} \\
& \quad+\nu\left(\mathbb{E} \int_{t \wedge \tau}^{T \wedge \tau} e^{\lambda s+\mu A_{s}}\left|\delta Y_{s}\right|^{2} d s+\mathbb{E} \int_{t \wedge \tau}^{T \wedge \tau} e^{\lambda s+\mu A_{s}}\left|\delta Y_{s}\right|^{2} d A_{s}\right) \\
& \quad+\frac{1}{2} \mathbb{E} \int_{t \wedge \tau}^{T \wedge \tau} e^{\lambda s+\mu A_{s}}\left(\left|\delta Z_{s}\right|^{2}+\left.|| \delta U_{s}\right|^{2}\right) d s \\
& \leq \mathbb{E} e^{\lambda(T \wedge \tau)+\mu A_{T \wedge \tau}}\left|\delta Y_{T}\right|^{2} .
\end{aligned}
$$

First, $\delta Y_{t}=0$ on the set $\{t \geq \tau\}$. On the other hand, we could prove similarly that

$$
\mathbb{E} e^{\lambda^{\prime}(t \wedge \tau)+\mu A_{t \wedge \tau}}\left|\delta Y_{t}\right|^{2} \leq \mathbb{E} e^{\lambda^{\prime}(T \wedge \tau)+\mu A_{T \wedge \tau}}\left|\delta Y_{T}\right|^{2}
$$

for $\lambda>\lambda^{\prime}>2 \alpha+4 K^{2}$. Then for $t \leq T \leq \tau$,

$$
\mathbb{E} e^{\lambda^{\prime} t+\mu A_{t}}\left|\delta Y_{t}\right|^{2} \leq e^{\left(\lambda^{\prime}-\lambda\right) T} \mathbb{E} e^{\lambda T+\mu A_{T}}\left|\delta Y_{T}\right|^{2} \leq C e^{\left(\lambda^{\prime}-\lambda\right) T} .
$$

The right-hand side goes to 0 as $T$ tend to $+\infty$.

It follows that $(\delta Y, \delta Z, \delta U)=(0,0,0)$.

Existence. In view of Theorem 3.3, by using the BSDE with data $\left(\xi_{n}, \square_{[0, \tau]} f, \square_{[0, \tau]} g, A\right)$, for each $n \geq 0$, we construct the sequence $\left(Y_{t}^{n}, Z_{t}^{n}, U_{t}^{n}\right)_{0 \leq t \leq T}$ solution of the following GBSDEL:

$$
\begin{aligned}
& Y_{t}^{n}= \xi_{n}+\int_{t \wedge \tau}^{n \wedge \tau} f\left(s, Y_{s^{-}}^{n}, Z_{s}^{n}, U_{s}^{n}\right) d s+\int_{t \wedge \tau}^{n \wedge \tau} g\left(s, Y_{s^{-}}^{n}\right) d A_{s}-\int_{t \wedge \tau}^{n \wedge \tau} Z_{s}^{n} d W_{s} \\
& \quad-\sum_{i=1}^{+\infty} \int_{t \wedge \tau}^{n \wedge \tau} U_{s}^{n(i)} d H_{s}^{(i)} \quad \forall 0 \leq t \leq n, \\
& Y_{t}^{n}=\xi_{t} \quad Z_{t}^{n}=\zeta_{t} \quad U_{t}^{n}=\rho_{t} \quad \forall t \geq n .
\end{aligned}
$$


We suppose that $Z_{t}^{n}=0, U_{t}^{n}=0$ for all $t>\tau$.

In fact, (3.46) is equivalent to

$$
\begin{aligned}
Y_{t}^{n}= & \xi+\int_{t}^{n} \mathbb{\square}_{[0, \tau]} f\left(s, Y_{s^{-}}^{n}, Z_{s}^{n}, U_{s}^{n}\right) d s+\int_{t}^{n} \mathbb{\square}_{[0, \tau]} g\left(s, Y_{s^{-}}^{n}\right) d A_{s}-\int_{t}^{n} Z_{s}^{n} d W_{s} \\
& -\sum_{i=1}^{+\infty} \int_{t}^{n} U_{s}^{n(i)} d H_{s}^{(i)} \quad \forall t \leq n .
\end{aligned}
$$

In the same way of Proposition 3.5, we can show that

$$
\begin{aligned}
\sup _{n \geq 0} \mathbb{E} & \left(\sup _{s \geq t} e^{\lambda(s \wedge \tau)+\mu A_{s \wedge \tau}}\left|Y_{s \wedge \tau}^{n}\right|^{2}+\int_{t \wedge \tau}^{\tau} e^{\lambda r+\mu A_{r}}\left|Y_{r}^{n}\right|^{2} d A_{r}\right. \\
& \left.+\int_{t \wedge \tau}^{\tau} e^{\lambda r+\mu A_{r}}\left(\left|Y_{r}^{n}\right|^{2}+\left|Z_{r}^{n}\right|^{2}+\left.|| U_{r}^{n}\right|^{2}\right) d r\right) \\
\leq & C \mathbb{E}\left(e^{\lambda \tau+\mu A_{\tau}}|\xi|^{2}+\int_{0}^{\tau} e^{\lambda r+\mu A_{r}}\left(|f(r, 0,0,0)|^{2} d r+|g(r, 0)|^{2} d A_{r}\right)\right) .
\end{aligned}
$$

We now prove that $\left(Y^{n}, Z^{n}, U^{n}\right)$ is a Cauchy sequence in the Banach space $\mathscr{H}_{\lambda, \mu}^{2}$.

We adopt the notations which are in the proof of Theorem 3.7.

(i) For $m \leq t \leq n$,

$$
\begin{aligned}
Y_{t}^{m}=\xi_{t} & =\mathbb{E}\left(\xi / \mathscr{F}_{n}\right)-\int_{t \wedge \tau}^{n \wedge \tau} \zeta_{s} d W_{s}-\sum_{i=1}^{+\infty} \int_{t \wedge \tau}^{n \wedge \tau} \rho_{s}^{(i)} d H_{s}^{(i)} \\
& =\mathbb{E}\left(\xi / \mathscr{F}_{n}\right)-\int_{t \wedge \tau}^{n \wedge \tau} Z_{s}^{m} d W_{s}-\sum_{i=1}^{+\infty} \int_{t \wedge \tau}^{n \wedge \tau} U_{s}^{m(i)} d H_{s}^{(i)}
\end{aligned}
$$

Then we have

$$
\begin{aligned}
\bar{Y}_{t}^{n, m}= & \int_{t \wedge \tau}^{n \wedge \tau} f\left(s, Y_{s^{-}}^{n}, Z_{s}^{n}, U_{s}^{n}\right) d s+\int_{t \wedge \tau}^{n \wedge \tau} g\left(s, Y_{s^{-}}^{n}\right) d A_{s}-\int_{t \wedge \tau}^{n \wedge \tau} \bar{Z}_{s}^{m, n} d W_{s} \\
& -\sum_{i=1}^{+\infty} \int_{t \wedge \tau}^{n \wedge \tau} \bar{U}_{s}^{n, m(i)} d H_{s}^{(i)} .
\end{aligned}
$$

If we apply Itô's formula, we obtain

$$
\begin{gathered}
\mathbb{E} e^{\lambda t+\mu A_{t}}\left|\bar{Y}_{t}^{n, m}\right|^{2}+\lambda \mathbb{E} \int_{t \wedge \tau}^{n \wedge \tau} e^{\lambda s+\mu A_{s}}\left|\bar{Y}_{s}^{n, m}\right|^{2} d s+\mu \mathbb{E} \int_{t \wedge \tau}^{n \wedge \tau} e^{\lambda s+\mu A_{s}}\left|\bar{Y}_{s}^{n, m}\right|^{2} d A_{s} \\
\quad+\mathbb{E} \int_{t \wedge \tau}^{n \wedge \tau} e^{\lambda s+\mu A_{s}}\left(\left|\bar{Z}_{s}^{n, m}\right|^{2}+\|\left.\bar{U}_{s}^{n, m}\right|^{2}\right) d s \\
=2 \mathbb{E} \int_{t \wedge \tau}^{n \wedge \tau} e^{\lambda s+\mu A_{s}} \bar{Y}_{s}^{n, m}\left(f\left(s, Y_{s}^{n}, Z_{s}^{n}, U_{s}^{n}\right) d s+g\left(s, Y_{s}^{n}\right) d A_{s}\right) .
\end{gathered}
$$


14 Generalized BSDE driven by a Lévy process

Since

$$
\begin{aligned}
\bar{Y}_{s}^{n, m} f\left(s, Y_{s}^{n}, Z_{s}^{n}, U_{s}^{n}\right)= & \bar{Y}_{s}^{n, m}\left(f\left(s, Y_{s}^{n}, Z_{s}^{n}, U_{s}^{n}\right)-f\left(s, \xi_{s}, Z_{s}^{n}, U_{s}^{n}\right)\right) \\
& +\bar{Y}_{s}^{n, m}\left(f\left(s, \xi, Z_{s}^{n}, U_{s}^{n}\right)-f\left(s, \xi_{s}, \zeta_{s}, \rho_{s}\right)\right) \\
& +\bar{Y}_{s}^{n, m} f\left(s, \xi_{s}, \zeta_{s}, \rho_{s}\right)
\end{aligned}
$$

and in view of (3.iii) and (3.iv), we get

$$
\begin{aligned}
2 \bar{Y}_{s}^{n, m} f\left(s, Y_{s}^{n}, Z_{s}^{n}, U_{s}^{n}\right) \leq & \left(1+2 \alpha+4 K^{2}\right)\left|\bar{Y}_{s}^{n, m}\right|^{2}+\frac{1}{2}\left(\left|\bar{Z}_{s}^{n, m}\right|^{2}+\left\|\bar{U}_{s}^{n, m}\right\|^{2}\right) \\
& +\left|f\left(s, \xi_{s}, \zeta_{s}, \rho_{s}\right)\right|^{2} .
\end{aligned}
$$

In the same way, by (3.v), we can write

$$
\bar{Y}_{s}^{n, m} g\left(s, Y_{s}^{n}\right) \leq \frac{\beta}{2}\left|\bar{Y}_{s}^{n, m}\right|^{2}+\frac{2}{|\beta|}\left|g\left(s, \xi_{s}\right)\right|^{2} .
$$

We plug (3.53) and (3.54) in (3.51) to obtain

$$
\begin{aligned}
& \mathbb{E} e^{\lambda t+\mu A_{t}}\left|\bar{Y}_{t}^{n, m}\right|^{2}+\left(\lambda-\left(1+2 \alpha+4 K^{2}\right)\right) \mathbb{E} \int_{t \wedge \tau}^{n \wedge \tau} e^{\lambda s+\mu A_{s}}\left|\bar{Y}_{s}^{n, m}\right|^{2} d s \\
& +(\mu-\beta) \mathbb{E} \int_{t \wedge \tau}^{n \wedge \tau} e^{\lambda s+\mu A_{s}}\left|\bar{Y}_{s}^{n, m}\right|^{2} d A_{s}+\frac{1}{2} \mathbb{E} \int_{t \wedge \tau}^{n \wedge \tau} e^{\lambda s+\mu A_{s}}\left(\left|\bar{Z}_{s}^{n, m}\right|^{2}+\| \bar{U}_{s}^{n, m}||^{2}\right) d s \\
& \leq \mathbb{E} \int_{m \wedge \tau}^{\tau} e^{\lambda s+\mu A_{s}}\left|f\left(s, \xi_{s}, \zeta_{s}, \rho_{s}\right)\right|^{2} d s+\left.\frac{2}{|\beta|}\left|\mathbb{E} \int_{m \wedge \tau}^{\tau} e^{\lambda s+\mu A_{s}}\right| g\left(s, \xi_{s}\right)\right|^{2} d A_{s} .
\end{aligned}
$$

We conclude that there exists $C>0$ such that

$$
\begin{array}{rl}
\sup _{m \leq t \leq n} & \mathbb{E} e^{\lambda t+\mu A_{t}}\left|\bar{Y}_{t}^{n, m}\right|^{2} \\
& +\mathbb{E} \int_{m \wedge \tau}^{n \wedge \tau} e^{\lambda s+\mu A_{s}}\left|\bar{Y}_{s}^{n, m}\right|^{2}\left(d s+d A_{s}\right) \\
& +\mathbb{E} \int_{m \wedge \tau}^{n \wedge \tau} e^{\lambda s+\mu A_{s}}\left(\left|\bar{Z}_{s}^{n, m}\right|^{2}+\left\|\bar{U}_{s}^{n, m}\right\|^{2}\right) d s \\
\leq & C\left(\mathbb{E} \int_{m \wedge \tau}^{\tau} e^{\lambda s+\mu A_{s}}\left|f\left(s, \xi_{s}, \zeta_{s}, \rho_{s}\right)\right|^{2} d s+\mathbb{E} \int_{m \wedge \tau}^{\tau} e^{\lambda s+\mu A_{s}}\left|g\left(s, \xi_{s}\right)\right|^{2} d A_{s}\right) .
\end{array}
$$

The last time of this inequality tends to 0 as $m$ goes to infinity.

(ii) For $t \leq m \leq n$, since

$$
\begin{aligned}
\bar{Y}_{m}^{n, m}= & \int_{m \wedge \tau}^{n \wedge \tau} f\left(s, Y_{s^{-}}^{n}, Z_{s}^{n}, U_{s}^{n}\right) d s-\int_{m \wedge \tau}^{n \wedge \tau} g\left(s, Y_{s^{-}}^{n}\right) d A_{s}-\int_{m \wedge \tau}^{n \wedge \tau} Z_{s}^{n} d W_{s} \\
& -\sum_{i=1}^{+\infty} \int_{m \wedge \tau}^{n \wedge \tau} U_{s}^{n(i)} d H_{s}^{(i)},
\end{aligned}
$$


we have

$$
\begin{aligned}
\bar{Y}_{t}^{n, m}= & \bar{Y}_{m}^{n, m}+\int_{t \wedge \tau}^{m \wedge \tau} \bar{f}_{s}^{n, m} d s+\int_{t \wedge \tau}^{m \wedge \tau} \bar{g}_{s}^{n, m} d A_{s}-\int_{t \wedge \tau}^{m \wedge \tau} \bar{Z}_{s}^{n, m} d W_{s} \\
& -\sum_{i=1}^{+\infty} \int_{t \wedge \tau}^{m \wedge \tau} \bar{U}_{s}^{n, m(i)} d H_{s}^{(i)} .
\end{aligned}
$$

An argument analogous to that used in the proof of Proposition 3.6 yields

$$
\begin{aligned}
\mathbb{E} e^{\lambda t+\mu A_{t}}\left|\bar{Y}_{t}^{n, m}\right|^{2}+\mathbb{E} \int_{t \wedge \tau}^{m \wedge \tau} e^{\lambda s+\mu A_{s}}\left|\bar{Y}_{s}^{n, m}\right|^{2} d s+\mathbb{E} \int_{t \wedge \tau}^{m \wedge \tau} e^{\lambda s+\mu A_{s}}\left|\bar{Y}_{s}^{n, m}\right|^{2} d A_{s} \\
\quad+\mathbb{E} \int_{t \wedge \tau}^{m \wedge \tau} e^{\lambda s+\mu A_{s}}\left(\left|\bar{Z}_{s}^{n, m}\right|^{2}+\left\|\bar{U}_{s}^{n, m}\right\|^{2}\right) d s \\
\leq C \mathbb{E} e^{\lambda m+\mu A_{m}}\left|\bar{Y}_{m}^{n, m}\right|^{2} .
\end{aligned}
$$

In view of (3.56), the right-hand side tends to 0 as $m$ goes to infinity, and one concludes that $\left(Y^{n}, Z^{n}, U^{n}\right)$ is a Cauchy sequence for the $\mathscr{H}_{\lambda, \mu}^{2}$ norm. Its limit is the solution of (3.4) and it satisfies (3.7).

\section{GBSDEL with a left-continuous coefficient}

In this section, we study the GBSDEL with continuous coefficient. We present a comparison theorem when the coefficient is uniformly Lipschitz and we prove existence of a solution when the coefficient is left continuous, increasing, and bounded.

To begin with, let us consider the GBSDEL:

$$
Y_{t}=\xi+\int_{t}^{T} f\left(s, Y_{s^{-}}, Z_{s}\right) d s+\int_{t}^{T} g\left(s, Y_{s^{-}}\right) d A_{s}-\int_{t}^{T} Z_{s} d W_{s}-\sum_{i=1}^{+\infty} \int_{t}^{T} U_{s}^{(i)} d H_{s}^{(i)} .
$$

We suppose that there exist $M, K>0$, and $\beta<0$, such that

(4.i) $\mathbb{E}|\xi|^{2}<+\infty$,

(4.ii) $f(\cdot, y, z)$ and $g(\cdot, y)$ are progressively measurable for all $(t, y, z)$,

(4.iii) $y \mapsto f(t, y, z)$ is left continuous and increasing such that

$$
|f(t, y, z)| \leq M \quad \forall(t, y, z)
$$

(4.iv) $\left|f(t, y, z)-f\left(t, y, z^{\prime}\right)\right| \leq K\left|z-z^{\prime}\right|$ for all $(t, y)$,

(4.v) $\left(y-y^{\prime}\right)\left(g(t, y)-g\left(t, y^{\prime}\right)\right) \leq \beta\left|y-y^{\prime}\right|^{2}$,

(4.vi) $y \mapsto g(t, y)$ is continuous such that $|g(t, y)| \leq K(1+|y|)$.

Let $\left(\xi^{i}, f^{i}, g^{i}, A\right)$ for $i=1,2$ be two sets of data, each satisfying the assumptions (4.i), (4.ii), (4.iv)-(4.vi), and

$$
\left|f(t, y, z)-f\left(t, y^{\prime}, z\right)\right| \leq K\left|y-y^{\prime}\right|
$$


For $i=1,2$, let $\left(Y^{i}, Z^{i}, U^{i}\right)$ denote a solution of the GBSDEL (4.1) with data $\left(\xi^{i}, f^{i}, g^{i}, A\right)$. The comparison theorem is not true in general case (see [3] for a counter-example).

Theorem 4.1. Suppose that $\xi^{1} \leq \xi^{2}, f^{1}(t, y, z) \leq f^{2}(t, y, z)$, and $g^{1}(t, y) \leq g^{2}(t, y)$ for all $(t, y, z) \in[0, T] \times \mathbb{R} \times \mathbb{R}, d \mathbb{P} \times d t$ a.s. Then $Y_{t}^{1} \leq Y_{t}^{2}$ for all $0 \leq t \leq T$ a.s.

Proof. Define

$$
\begin{aligned}
& \alpha_{t}= \begin{cases}\left(Y_{t}^{2}-Y_{t}^{1}\right)^{-1}\left(f^{1}\left(t, Y_{t}^{2}, Z_{t}^{1}\right)-f^{1}\left(t, Y_{t}^{1}, Z_{t}^{1}\right)\right) & \text { if } Y_{t}^{1} \neq Y_{t}^{2}, \\
0 & \text { if } Y_{t}^{1}=Y_{t}^{2},\end{cases} \\
& \beta_{t}= \begin{cases}\left(Z_{t}^{2}-Z_{t}^{1}\right)^{-1}\left(f^{1}\left(t, Y_{t}^{2}, Z_{t}^{2}\right)-f^{1}\left(t, Y_{t}^{2}, Z_{t}^{1}\right)\right) & \text { if } Z_{t}^{1} \neq Z_{t}^{2}, \\
0 & \text { if } Z_{t}^{1}=Z_{t}^{2},\end{cases} \\
& \gamma_{t}= \begin{cases}\left(Y_{t}^{2}-Y_{t}^{1}\right)^{-1}\left(g^{1}\left(t, Y_{t}^{2}\right)-g^{1}\left(t, Y_{t}^{1}\right)\right) & \text { if } Y_{t}^{1} \neq Y_{t}^{2}, \\
0 & \text { if } Y_{t}^{1}=Y_{t}^{2}\end{cases}
\end{aligned}
$$

three progressively measurable processes such that $\left|\alpha_{t}\right| \vee\left|\beta_{t}\right| \leq K$ and $\gamma_{t} \leq \beta$.

For $0 \leq s \leq t \leq T$, the SDE:

$$
\Gamma_{s, t}=1+\int_{s}^{t} \Gamma_{s, r} \alpha_{r} d r+\int_{s}^{t} \Gamma_{s, r} \beta_{r} d W_{r}+\int_{s}^{t} \Gamma_{s, r} \gamma_{r} d A_{r}
$$

has a unique solution, and we can write that

$$
\Gamma_{s, t}=\exp \left(\int_{s}^{t}\left(\alpha_{r}-\frac{\beta_{r}^{2}}{2}\right) d r+\int_{s}^{t} \beta_{r} d W_{r}+\int_{s}^{t} \gamma_{r} d A_{r}\right) .
$$

We denote by

$$
\begin{gathered}
\delta \xi=\xi^{2}-\xi^{1}, \quad \delta Y_{s}=Y_{s}^{2}-Y_{s}^{1}, \quad \delta Z_{s}=Z_{s}^{2}-Z_{s}^{1}, \quad \delta U_{s}=U_{s}^{2}-U_{s}^{1}, \\
\delta f_{s}=f^{2}\left(s, Y_{s}^{2}, Z_{s}^{2}\right)-f^{1}\left(s, Y_{s}^{2}, Z_{s}^{2}\right), \quad \delta g_{s}=g^{2}\left(s, Y_{s}^{2}\right)-g^{1}\left(s, Y_{s}^{2}\right) .
\end{gathered}
$$

In view of the above notations, we get

$$
\begin{aligned}
\delta Y_{t}= & \delta \xi+\int_{t}^{T}\left(\alpha_{r} \delta y_{r}+\beta \delta Z_{r}+\delta f_{r}\right) d r \\
& +\int_{t}^{T}\left(\gamma_{r} \delta Y_{r}+\delta g_{r}\right) d A_{r}-\int_{t}^{T} \delta Z_{r} d W_{r}-\sum_{i=1}^{\infty} \int_{t}^{T} \delta U_{r}^{(i)} d H_{r}^{(i)} .
\end{aligned}
$$

By the integration-by-part formula, we have

$$
\begin{aligned}
\delta Y_{t} \Gamma_{s, t}= & \delta Y_{s}+\int_{s}^{t} \Gamma_{s, r}\left(\delta Y_{r} \beta_{r}+\delta Z_{r}\right) d W_{r}+\sum_{i=1}^{\infty} \int_{t}^{T} \Gamma_{s, r} \delta U_{r}^{(i)} d H_{r}^{(i)} \\
& -\int_{s}^{t} \Gamma_{s, r} \delta f_{r} d r-\int_{s}^{t} \Gamma_{s, r} \delta g_{r} d A_{r} .
\end{aligned}
$$


In particular for $t=T$,

$$
\delta Y_{s}=\mathbb{E}\left(\delta \xi \Gamma_{s, T}+\int_{s}^{T} \Gamma_{s, r}\left(\delta f_{r} d r+\delta g_{r} d A_{r}\right) / \mathscr{F}_{s}\right) \geq 0
$$

The result is as follows.

Theorem 4.2. Under the assumptions (4.i)-(4.vi), there exists a unique process $\left(Y_{t}, Z_{t}\right.$, $\left.U_{t}\right)_{0 \leq t \leq T}$ solution of (4.1).

We construct the sequence $\left(f_{n}\right)_{n \geq 1}$ such that

$$
f_{n}(s, y, z)=n \int_{y-1 / n}^{y} f(s, x, z) d x
$$

which verifies the following properties (see [5]).

$(4 . i)^{\prime}$ For all $n, f_{n}(t, \cdot, y, z)$ is progressively measurable.

(4.ii) For all $n, \exists K_{n}>0,\left|f_{n}(t, y, z)-f_{n}\left(t, y^{\prime}, z\right)\right| \leq K_{n}\left|y-y^{\prime}\right|$.

(4.iii) $)^{\prime}\left|f_{n}(t, y, z)-f_{n}\left(t, y, z^{\prime}\right)\right| \leq K\left|z-z^{\prime}\right|$.

(4.iv) $\exists M>0$ such that $\sup _{n \geq 1} \sup _{0 \leq y \leq T} \sup _{(y, z) \in \mathbb{R} \times \mathbb{R}}\left|f_{n}(s, y, z)\right| \leq M$.

$(4 . \mathrm{v})^{\prime}\left(f_{n}(t, y, z)\right)_{n \geq 1}$ is increasing for all $(t, y, z)$.

(4.vi)' For all $n, y \mapsto f_{n}(s, y, z)$ is increasing.

(4.vii)' If $y_{n} \uparrow y$, then $\lim _{n \rightarrow+\infty} f_{n}\left(s, y_{n}, z\right)=f(s, y, z)$.

For all $n \geq 1$, there exists $\left(Y_{t}^{n}, Z_{t}^{n}, U_{t}^{n}\right)$ solution of the GBSDEL:

$$
Y_{t}^{n}=\xi+\int_{t}^{T} f_{n}\left(s, Y_{s^{-}}^{n}, Z_{s}^{n}\right) d s+\int_{t}^{T} g\left(s, Y_{s^{-}}^{n}\right) d A_{s}-\int_{t}^{T} Z_{s}^{n} d W_{s}-\sum_{i=1}^{+\infty} \int_{t}^{T} U_{s}^{n(i)} d H_{s}^{(i)} .
$$

Proposition 4.3. There exists $C>0$, a constant only depending on $\xi, T$, and $M$ such that

$$
\sup _{n \geq 1} \mathbb{E}\left(\sup _{0 \leq t \leq T}\left|Y_{t}^{n}\right|^{2}+\int_{0}^{T}\left|Y_{s}^{n}\right|^{2} d A_{s}+\int_{0}^{T}\left(\left|Z_{s}^{n}\right|^{2}+\left\|U_{s}^{n}\right\|^{2}\right) d s\right) \leq C .
$$

Proof. From Itô's formula, we have

$$
\begin{aligned}
\mathbb{E}\left|Y_{t}^{n}\right|^{2}+\mathbb{E} \int_{t}^{T}\left(\left|Z_{s}^{n}\right|^{2}+\| U_{s}^{n}||^{2}\right) d s \\
=\mathbb{E}|\xi|^{2}+2 \mathbb{E} \int_{t}^{T} Y_{s}^{n} f_{n}\left(s, Y_{s}^{n}, Z_{s}^{n}\right) d s+2 \mathbb{E} \int_{t}^{T} Y_{s}^{n} g\left(s, Y_{s}^{n}\right) d A_{s} \\
\leq \mathbb{E}|\xi|^{2}+2 \mathbb{E} \sup _{0 \leq s \leq T}\left|f_{n}\left(s, Y_{s}^{n}, Z_{s}^{n}\right)\right| \int_{t}^{T}\left|Y_{s}^{n}\right| d s+2 \mathbb{E} \int_{t}^{T}\left(\frac{\beta}{2}\left|Y_{s}^{n}\right|^{2}+\frac{2}{|\beta|}|g(s, 0)|^{2}\right) d A_{s} \\
\leq \mathbb{E}|\xi|^{2}+T \mathbb{E} \sup _{0 \leq s \leq T}\left|f_{n}\left(s, Y_{s}^{n}, Z_{s}^{n}\right)\right|^{2}+\frac{4}{|\beta|} \mathbb{E} \int_{0}^{T}|g(s, 0)|^{2} d A_{s} \\
\quad+\mathbb{E} \int_{t}^{T}\left|Y_{s}^{n}\right|^{2} d s+\beta \mathbb{E} \int_{t}^{T}\left|Y_{s}^{n}\right|^{2} d A_{s} .
\end{aligned}
$$


By the condition $(4 . v)^{\prime}$, we get for all $0 \leq t \leq T$,

$$
\begin{gathered}
\mathbb{E}\left|Y_{t}^{n}\right|^{2}+|\beta| \mathbb{E} \int_{t}^{T}\left|Y_{s}^{n}\right|^{2} d A_{s}+\mathbb{E} \int_{t}^{T}\left(\left|Z_{s}^{n}\right|^{2}+\|\left. U_{s}^{n}\right|^{2}\right) d s \\
\leq \mathbb{E}|\xi|^{2}+M^{2}\left(T+\frac{4 \mathbb{E}\left(A_{T}\right)}{|\beta|}\right)+\mathbb{E} \int_{t}^{T}\left|Y_{s}^{n}\right|^{2} d s .
\end{gathered}
$$

The result follows from this inequality, the Gronwall formula, and the Burkhölder-DavisGundy inequality.

Proof of Theorem 4.2. The sequence $\left(f_{n}\right)_{n \geq 1}$ is increasing, the comparison theorem implies that for all $t,\left(Y_{t}^{n}\right)_{n \geq 1}$ is increasing. Moreover, $\operatorname{Esup}_{0 \leq t \leq T}\left|Y_{t}^{n}\right|^{2} \leq C$, then $Y^{n} \uparrow Y$. Using Fatou's lemma, we obtain $\mathbb{E} \sup _{0 \leq t \leq T}\left|Y_{t}\right|^{2} \leq C$. On the other hand, $\mathbb{E} \int_{0}^{T}\left|Y_{t}^{n}\right|^{2} d t \leq C$, then, by Lebesgue's dominated convergence theorem, we deduce that

$$
\mathbb{E} \int_{0}^{T}\left|Y_{t}^{n}-Y_{t}\right|^{2} d t \longrightarrow 0 \quad \text { as } n \longrightarrow \infty
$$

Let $n \geq m \geq 1$, we denote by

$$
\bar{Y}_{s}^{n, m}=Y_{s}^{n}-Y_{s}^{m}, \quad \bar{Z}_{s}^{n, m}=Z_{s}^{n}-Z_{s}^{m}, \quad \bar{U}_{s}^{n, m}=U_{s}^{n}-U_{s}^{m} .
$$

Using Itô's formula, we get

$$
\begin{aligned}
\mathbb{E}\left|\bar{Y}_{t}^{n, m}\right|^{2}+\mathbb{E} \int_{t}^{T}\left(\left|\bar{Z}_{s}^{n, m}\right|^{2}+\| \bar{U}_{s}^{n, m}||^{2}\right) d s \\
\leq 2 \mathbb{E} \int_{t}^{T}\left|\bar{Y}_{s}^{n, m}\right|\left|f_{n}\left(s, Y_{s}^{n}, Z_{s}^{n}\right)-f_{m}\left(s, Y_{s}^{m}, Z_{s}^{m}\right)\right| d s \\
\quad+2 \mathbb{E} \int_{t}^{T} \bar{Y}_{s}^{n, m}\left(g\left(s, Y_{s}^{n}\right)-g\left(s, Y_{s}^{m}\right)\right) d A_{s} \\
\leq 4 M \sqrt{T}\left(\mathbb{E} \int_{0}^{T}\left|\bar{Y}_{s}^{n, m}\right|^{2} d s\right)^{1 / 2} .
\end{aligned}
$$

The right-hand side goes to 0 as $m$ and $n$ tend to infinity.

Now, we can show that $\mathbb{E} \sup _{0 \leq t \leq T}\left|\bar{Y}_{t}^{n, m}\right|^{2} \rightarrow 0$.

If we apply Itô's formula, we obtain

$$
\begin{aligned}
\left|\bar{Y}_{t}^{n, m}\right|^{2} & +\int_{t}^{T}\left(\left|\bar{Z}_{s}^{n, m}\right|^{2}+\left\|\bar{U}_{s}^{n, m}\right\|^{2}\right) d s \\
= & 2 \int_{t}^{T} \bar{Y}_{s}^{n, m}\left(f_{n}\left(s, Y_{s^{-}}^{n}, Z_{s}^{n}\right)-f_{m}\left(s, Y_{s^{-}}^{m}, Z_{s}^{m}\right)\right) d s \\
& +2 \int_{t}^{T} \bar{Y}_{s}^{n, m}\left(g\left(s, Y_{s^{-}}^{n}\right)-g\left(s, Y_{s^{-}}^{m}\right)\right) d A_{s} \\
& -2 \int_{t}^{T} \bar{Y}_{s}^{n, m} \bar{Z}_{s}^{n, m} d W_{s}-2 \sum_{i=1}^{+\infty} \int_{t}^{T} \bar{Y}_{s}^{n, m} \bar{U}_{s}^{n, m(i)} d H_{s}^{(i)} .
\end{aligned}
$$


By Burkhölder-Davis-Gundy inequality, we can write

$$
\begin{aligned}
& \sup _{0 \leq t \leq T}\left|\bar{Y}_{t}^{n, m}\right|^{2}+\mathbb{E} \int_{0}^{T}\left(\left|\bar{Z}_{s}^{n, m}\right|^{2}+\left\|\bar{U}_{s}^{n, m}\right\|^{2}\right) d s \\
& \quad \leq 2 M \sqrt{T}\left(\mathbb{E} \int_{0}^{T}\left|\bar{Y}_{s}^{n, m}\right|^{2} d s\right)^{1 / 2}+\frac{1}{2} \mathbb{E} \sup _{0 \leq t \leq T}\left|\bar{Y}_{t}^{n, m}\right|^{2}+4 \mathbb{E} \int_{0}^{T}\left(\left|\bar{Z}_{s}^{n, m}\right|^{2}+\left\|\bar{U}_{s}^{n, m}\right\|^{2}\right) d s .
\end{aligned}
$$

Then $\mathbb{E} \sup _{0 \leq t \leq T}\left|\bar{Y}_{t}^{n, m}\right|^{2} \leq C\left(E \int_{0}^{T}\left|\bar{Y}_{s}^{n, m}\right|^{2} d s\right)^{1 / 2}$. The right-hand side goes to 0 as $m$ and $n$ tend to infinity.

In conclusion, $\left(Y^{n}, Z^{n}, U^{n}\right)$ is a Cauchy sequence for the $\mathscr{H}^{2}$ norm.

It remains to show that $\left(Y_{t}, Z_{t}, U_{t}\right)=\lim _{n \rightarrow \infty}\left(Y_{t}^{n}, Z_{t}^{n}, U_{t}^{n}\right)$ is a solution of (4.1).

First, there exists a subsequence $\left(Y^{n}, Z^{n}\right) \rightarrow(Y, Z) d t \times d \mathbb{P}$ a.s. Using $(4 . v i)^{\prime}$, for almost all $\omega$, we have

$$
f_{n}\left(t, Y_{t}^{n}, Z_{t}\right) \longrightarrow f\left(t, Y_{t}, Z_{t}\right) d t \quad \text { a.e. }
$$

So,

$$
\left|f_{n}\left(t, Y_{t}^{n}, Z_{t}^{n}\right)-f\left(t, Y_{t}, Z_{t}\right)\right| \leq K\left|Z_{t}^{n}-Z_{t}\right|+\left|f_{n}\left(t, Y_{t}^{n}, Z_{t}\right)-f\left(t, Y_{t}, Z_{t}\right)\right|
$$

Then, for almost all $\omega, f_{n}\left(t, Y_{t}^{n}, Z_{t}^{n}\right) \stackrel{n \rightarrow \infty}{\longrightarrow} f\left(t, Y_{t}, Z_{t}\right) d t$ a.s.

Since $\sup _{n \geq 1}\left|f_{n}\left(t, Y_{t}^{n}, Z_{t}^{n}\right)\right| \leq M$, by Lebesgue's dominate convergence theorem for almost all $\omega$, we get

$$
\int_{0}^{T} f_{n}\left(s, Y_{s}^{n}, Z_{s}^{n}\right) d s \longrightarrow \int_{0}^{T} f\left(s, Y_{s}, Z_{s}\right) d s \quad \text { as } n \longrightarrow \infty
$$

In the same way, combining Fatou's lemma and Lebesgue's dominate convergence theorem shows that

$$
\int_{0}^{T} g\left(s, Y_{s}^{n}\right) d A_{s} \longrightarrow \int_{0}^{T} g\left(s, Y_{s}\right) d A_{s} \quad \text { as } n \longrightarrow \infty .
$$

We note by the Burkhölder-Davis-Gundy inequality that

$$
\begin{gathered}
\mathbb{E} \sup _{0 \leq t \leq T}\left|\int_{t}^{T} Z_{s}^{n} d W_{s}-\int_{t}^{T} Z_{s} d W_{s}\right|^{2} \leq C \mathbb{E} \int_{0}^{T}\left|Z_{s}^{n}-Z_{s}\right|^{2} d s, \\
\mathbb{E} \sup _{0 \leq t \leq T}\left|\sum_{i=1}^{\infty} \int_{t}^{T} U_{s}^{n(i)} d H_{s}^{(i)}-\sum_{i=1}^{\infty} \int_{t}^{T} U_{s}^{(i)} d H_{s}^{(i)}\right|^{2} \leq C \mathbb{E} \int_{0}^{T}\left\|U_{s}^{n}-U_{s}\right\|^{2} d s .
\end{gathered}
$$


Then for a subsequence

$$
\begin{gathered}
\sup _{0 \leq t \leq T}\left|\int_{t}^{T} Z_{s}^{n} d W_{s}-\int_{t}^{T} Z_{s} d W_{s}\right| \stackrel{n \rightarrow \infty}{\longrightarrow} 0, \\
\sup _{0 \leq t \leq T}\left|\sum_{i=1}^{\infty} \int_{t}^{T} U_{s}^{n(i)} d H_{s}^{(i)}-\sum_{i=1}^{\infty} \int_{t}^{T} U_{s}^{(i)} d H_{s}^{(i)}\right| \stackrel{n \rightarrow \infty}{\longrightarrow} 0 .
\end{gathered}
$$

To finish with, we write

$$
\begin{aligned}
\mid Y_{t}-\xi & -\int_{t}^{T} f\left(s, Y_{s^{-}}, Z_{s}\right) d s-\int_{t}^{T} g\left(s, Y_{s^{-}}\right) d A_{s}+\int_{t}^{T} Z_{s} d W_{s}+\sum_{i=1}^{\infty} \int_{t}^{T} U_{s}^{(i)} d H_{s}^{(i)} \mid \\
\leq & \sup _{0 \leq t \leq T}\left|Y_{t}^{n}-Y_{t}\right|+\left|\int_{0}^{T} f_{n}\left(s, Y_{s^{-}}^{n}, Z_{s}^{n}\right)-f\left(s, Y_{s^{-}}, Z_{s}\right) d s\right| \\
& +\left|\int_{0}^{T} g\left(s, Y_{s^{-}}^{n}\right)-g\left(s, Y_{s^{-}}\right) d A_{s}\right| \\
& +\sup _{0 \leq t \leq T}\left|\int_{t}^{T} Z_{s}^{n} d W_{s}-\int_{t}^{T} Z_{s} d W_{s}\right|+\sup _{0 \leq t \leq T}\left|\sum_{i=1}^{\infty} \int_{t}^{T} U_{s}^{n(i)} d H_{s}^{(i)}-\sum_{i=1}^{\infty} \int_{t}^{T} U_{s}^{(i)} d H_{s}^{(i)}\right| .
\end{aligned}
$$

The right-hand side goes to 0 as $n$ tends to infinity.

We conclude that for all $t \in[0, T]$,

$$
Y_{t}=\xi+\int_{t}^{T} f\left(s, Y_{s^{-}}, Z_{s}\right) d s+\int_{t}^{T} g\left(s, Y_{s^{-}}\right) d A_{s}-\int_{t}^{T} Z_{s} d W_{s}-\sum_{i=1}^{+\infty} \int_{t}^{T} U_{s}^{(i)} d H_{s}^{(i)} \mathbb{P} \quad \text { a.s. }
$$

This completes the proof of the theorem.

Remark 4.4. We obtain the same result if we suppose that

(i) $f$ is right continuous, decreasing, and bounded,

(ii) $f$ is continuous with linear growth in $y$ independent of $z$ (see [13] for approximation).

\section{Application to PDIE}

In this section, we study the link between generalized BSDE driven by Lévy process and a class of partial differential integral equations with Neumann boundary condition. We suppose that the process $L$ has bounded jump (without lost of generality, we suppose that $\left.\sup _{t}\left|\Delta L_{t}\right| \leq 1\right)$. Then, for all $p=1,2,3, \ldots, \mathbb{E}\left|L_{t}\right|^{p}<\infty$ (see [23, Theorem 34, page 25]), and by Lévy decomposition theorem (see [23, page 31]),

$$
L_{t}=b t+\int_{(|z|<1)} z\left(N_{t}(\cdot, d z)-t v(d z)\right)
$$


where $N_{t}(\omega, d z)$ denotes the random measure such that $\int_{\Lambda} N_{t}(\cdot, d z)$ is a Poisson process with parameter $\nu(\Lambda)$ for all set $\Lambda(0 \notin \bar{\Lambda})$.

Let $\Theta=(-l, l)$, and $n:[-l, l] \rightarrow \mathbb{R}$ such that $n(-l)=1$ and $n(l)=-1$.

Let us consider the two bounded coefficients $c, \sigma: \mathbb{R} \rightarrow \mathbb{R}$, satisfying for some $K, \kappa>0$, the following properties:

(5.i) $|c(x)|+|\sigma(x)| \leq \kappa$ for all $x \in \bar{\Theta}$,

(5.ii) $\left|c(x)-c\left(x^{\prime}\right)\right|+\left|\sigma(x)-\sigma\left(x^{\prime}\right)\right| \leq K\left|x-x^{\prime}\right|$ for every $x, x^{\prime} \in \bar{\Theta}$,

(5.iii) $x+z c(x) \rrbracket_{(|z| \leq 1)} \in \bar{\Theta}$ for every $x \in \bar{\Theta}$ and $z \in \mathbb{R}$,

(5.iv) $c(x)=c(\operatorname{pr}(x))$ for all $x \in \mathbb{R}$,

where $\operatorname{pr}(\cdot)$ denotes the orthogonal projection on the closure $\bar{\Theta}$.

Consider the reflected SDE:

$$
\begin{gathered}
X_{t}=x+\int_{0}^{t} \sigma\left(X_{s}\right) d W_{s}+\int_{0}^{t} c\left(X_{s^{-}}\right) d L_{s}+\eta_{t}, \\
\eta_{t}=\int_{0}^{t} n\left(X_{s}\right) d|\eta|_{s} \quad \text { with }|\eta|_{t}=\int_{0}^{t} \mathbb{q}_{\left(X_{s} \in \partial \Theta\right)} d|\eta|_{s} .
\end{gathered}
$$

In [15], Menaldi and Robin prove that under the assumptions (5.i)-(5.iv), there exists a unique pair of progressively measurable processes $(X, \eta)$ that verifies $(5.2)$, and for every progressively measurable process $V$ which is right continuous having left-hand limits and taking values in $\bar{\Theta}$, we have

$$
\int_{0}^{T}\left(X_{t}-V_{t}\right) d|\eta|_{t} \geq 0 .
$$

Let $u=u(t, x)$ be the solution of the following PDIE:

$$
\begin{gathered}
\partial_{t} u(t, x)+\bar{c}(x) \partial_{x} u(t, x)+\frac{1}{2} \sigma^{2}(x) \partial_{x x}^{2} u(t, x)+\int_{\mathbb{R}} u^{1}(t, x, z) v(d z) \\
+f\left(t, x, u(t, x), \sigma(x) \partial_{x} u(t, x),\left(u^{(i)}(t, x)\right)_{i=1}^{\infty}\right)=0 \quad \forall(t, x) \in[0, T) \times \Theta, \\
n(x) \partial_{x} u(t, x)+g(t, x, u(t, x))=0 \quad \forall(t, x) \in[0, T) \times\{-l ; l\}, \\
u(T, x)=h(x) \quad \forall x \in \bar{\Theta},
\end{gathered}
$$

where

(i) $\bar{c}(x)=m_{1} c(x)$,

(ii) $u^{1}(t, x, z)=u(t, x+c(x) z)-u(t, x)-\partial_{x} u(t, x) c(x) z$,

(iii) $u^{(1)}(t, x)=\left(m_{2}\right)^{1 / 2} c(x) \partial_{x} u(t, x)+\int_{\mathbb{R}} u^{1}(t, x, z) p_{1}(z) v(d z)$ and for $i \geq 2, u^{(i)}(t, x)=$ $\int_{\mathbb{R}} u^{1}(t, x, z) p_{i}(z) v(d z)$.

Consider the GBSDEL:

$$
\begin{aligned}
Y_{t}= & h\left(X_{T}\right)+\int_{t}^{T} f\left(s, X_{s^{-}}, Y_{s^{-}}, Z_{s}, U_{s}\right) d s+\int_{t}^{T} g\left(s, X_{s^{-}}, Y_{s^{-}}\right) d|\eta|_{s}-\int_{t}^{T} Z_{s} d W_{s} \\
& -\sum_{i=1}^{\infty} \int_{t}^{T} U_{s}^{(i)} d H_{s}^{(i)} .
\end{aligned}
$$


Suppose that the function $u \in \mathscr{C}^{1,2}([0, T] \times \mathbb{R})$, and is such that $\partial_{x} u$ and $\partial_{x x}^{2} u$ are bounded by polynomial function of $x$. Then we have the following theorem.

Theorem 5.1. The process $\left(Y, Z, U=\left(U^{(i)}\right)_{i=1}^{\infty}\right)$ given by

$$
\begin{gathered}
Y_{t}=u\left(t, X_{t}\right), \\
Z_{t}=\sigma\left(X_{t}\right) \partial_{x} u\left(t, X_{t}\right), \\
U_{t}^{(1)}=\int_{\mathbb{R}} u^{1}\left(t, X_{t^{-}}, z\right) p_{1}(z) v(d z)+\left(m_{2}\right)^{1 / 2} c\left(X_{t^{-}}\right) \partial_{x} u\left(t, X_{t^{-}}\right), \\
U_{t}^{(i)}=\int_{\mathbb{R}} u^{1}\left(t, X_{t^{-}}, z\right) p_{i}(z) v(d z) \quad \text { for } i \geq 2
\end{gathered}
$$

is solution of (5.5).

Proof. Applying Itô's formula to $u\left(s, X_{s}\right)$ from $s=t$ to $s=T$,

$$
\begin{aligned}
u\left(T, X_{T}\right)= & u\left(t, X_{t}\right)+\int_{t}^{T} \partial_{s} u\left(s, X_{s}\right) d s+\int_{t}^{T} n\left(X_{s}\right) \partial_{x} u\left(s, X_{s}\right) d|\eta|_{s} \\
& +\frac{1}{2} \int_{t}^{T} \sigma^{2}\left(X_{s}\right) \partial_{x x}^{2} u\left(s, X_{s}\right) d s+\int_{t}^{T} \sigma\left(X_{s}\right) \partial_{x} u\left(s, X_{s}\right) d W_{s} \\
& +\int_{t}^{T} c\left(X_{s^{-}}\right) \partial_{x} u\left(s, X_{s^{-}}\right) d L_{s} \\
& +\sum_{t<s \leq T} u\left(s, X_{s}\right)-u\left(s, X_{s^{-}}\right)-\partial_{x} u\left(s, X_{s^{-}}\right) \Delta X_{s} .
\end{aligned}
$$

If we apply [17, Lemma 5] to

$$
h(s, z)=u\left(s, X_{s^{-}}+c\left(X_{s^{-}}\right) z\right)-u\left(s, X_{s^{-}}\right)-\partial_{x} u\left(s, X_{s^{-}}\right) c\left(X_{s^{-}}\right) z,
$$

we have

$$
\begin{aligned}
\sum_{t<s \leq T} u\left(s, X_{s}\right)-u\left(s, X_{s^{-}}\right)-\partial_{x} u\left(s, X_{s^{-}}\right) \Delta X_{s} \\
\quad=\sum_{i=1}^{\infty} \int_{t}^{T} \int_{\mathbb{R}} u^{1}\left(s, X_{s^{-}}, z\right) p_{i}(z) v(d z) d H_{s}^{(i)}+\int_{t}^{T} \int_{\mathbb{R}} u^{1}\left(s, X_{s^{-}}, z\right) v(d z) d s .
\end{aligned}
$$

Since $H_{s}^{(1)}=\left(m_{2}\right)^{-1 / 2}\left(L_{s}-m_{1} s\right)$ and $\Delta X_{s}=c\left(X_{s^{-}}\right) \Delta L_{s}$ a.s for all $t<s \leq T$ (see [23, Theorem 12, page 60]). Substituting (5.9) into (5.7) to obtain

$$
\begin{aligned}
u\left(t, X_{t}\right)= & h\left(X_{T}\right)-\int_{t}^{T}\left(\partial_{s} u\left(s, X_{s}\right)+\bar{c}\left(X_{s}\right) \partial_{x} u\left(s, X_{s}\right)+\frac{1}{2} \sigma^{2}\left(X_{s}\right) \partial_{x x}^{2} u\left(s, X_{s}\right)\right) d s \\
& -\int_{t}^{T} \int_{\mathbb{R}} u^{1}\left(s, X_{s^{-}}, z\right) \nu(d z) d s-\int_{t}^{T} n\left(X_{s}\right) \partial_{s} u\left(s, X_{s}\right) \rrbracket_{\left(X_{s} \in \partial \Theta\right)} d|\eta|_{s}
\end{aligned}
$$




$$
\begin{aligned}
& -\int_{t}^{T} \sigma\left(X_{s}\right) \partial_{x} u\left(s, X_{s}\right) d W_{s}-\sum_{i=2}^{\infty} \int_{t}^{T} \int_{\mathbb{R}} u^{1}\left(s, X_{s^{-}}, z\right) p_{i}(z) v(d z) d H_{s}^{(i)} \\
& -\int_{t}^{T}\left(\int_{\mathbb{R}} u^{1}\left(s, X_{s^{-}}, z\right) p_{1}(z) v(d z)+\left(m_{2}\right)^{1 / 2} c\left(X_{s^{-}}\right) \partial_{x} u\left(s, X_{s^{-}}\right)\right) d H_{s}^{(1)},
\end{aligned}
$$

from which we get the result of the theorem.

We next consider some examples of PDIEs.

Example 5.2. Assume that $\nu(d x)=\sum_{i=1}^{\infty} \alpha_{i} \delta_{\beta_{i}}(d x)$, where $\alpha_{i}>0$ and $\delta_{\beta_{i}}(d x)$ denotes the positive point mass measure at $\beta_{i} \in \mathbb{R}$ of size one. Assume that $\sum_{i=1}^{\infty} \alpha_{i}\left|\beta_{i}\right|^{2}<\infty$. Then the process $L$ can be writing $L_{t}=b t+\sum_{i=1}^{\infty} \beta_{i}\left(N_{t}^{(i)}-\alpha_{i} t\right)$, where $\left(N^{(i)}\right)_{i=1}^{\infty}$ is a sequence of independent Poisson processes with parameters $\left(\alpha_{i}\right)_{i=1}^{\infty}$.

Recall that $H_{t}^{(1)}=\sum_{i=1}^{\infty}\left(\beta_{i} / \sqrt{\alpha_{i}}\right)\left(N_{t}^{(i)}-\alpha_{i} t\right)$ and $H_{t}^{(i)}=0$ for all $i \geq 2$ (see [17]).

Let $(Y, Z, U)$ be the unique solution of the following GBSDEL:

$$
\begin{aligned}
Y_{t}= & h\left(X_{T}\right)+\int_{t}^{T} f\left(s, X_{s^{-}}, Y_{s^{-}}, Z_{s}\right) d s+\int_{t}^{T} g\left(s, X_{s^{-}}, Y_{s^{-}}\right) d|\eta|_{s}-\int_{t}^{T} Z_{s} d W_{s} \\
& -\sum_{i=1}^{\infty} \int_{t}^{T} U_{s}^{(i)} d\left(N_{s}^{(i)}-\alpha_{i} s\right) .
\end{aligned}
$$

Then

$$
\begin{gathered}
Y_{t}=u\left(t, X_{t}\right), \\
Z_{t}=\sigma\left(X_{t}\right) \partial_{x} u\left(t, X_{t}\right), \\
U_{t}^{(1)}=\alpha_{1} u^{1}\left(t, X_{t^{-}}, \beta_{1}\right) p_{1}\left(\beta_{1}\right)+\left(\sum_{i=1}^{\infty} \alpha_{i}\left|\beta_{i}\right|^{2}\right)^{1 / 2} c\left(X_{t^{-}}\right) \partial_{x} u\left(t, X_{t^{-}}\right), \\
U_{t}^{(i)}=\alpha_{i} u^{1}\left(t, X_{t^{-}}, \beta_{i}\right) p_{1}\left(\beta_{i}\right) \quad \text { for } i \geq 2,
\end{gathered}
$$

where the function $u$ supposed in $\mathscr{C}^{1,2}([0, T] \times \mathbb{R})$ is such that $\partial_{x} u$ and $\partial_{x x}^{2} u$ are bounded by polynomial function of $x$, and it verifies the following PDIE:

$$
\begin{gathered}
\partial_{t} u(t, x)+\bar{c}(x) \partial_{x} u(t, x)+\frac{1}{2} \sigma^{2}(x) \partial_{x x}^{2} u(t, x)+\sum_{i=1}^{\infty} \alpha_{i} u^{1}\left(t, x, \beta_{i}\right) \\
+f\left(t, x, u(t, x), \sigma(x) \partial_{x} u(t, x)\right)=0 \quad \forall(t, x) \in[0, T) \times \Theta \\
n(x) \partial_{x} u(t, x)+g(t, x, u(t, x))=0 \quad \forall(t, x) \in[0, T) \times\{-l ; l\} \\
u(T, x)=h(x) \quad \forall x \in \bar{\Theta} .
\end{gathered}
$$


Example 5.3. We suppose that $L_{t}=N_{t}-\lambda t$, then the GBSDEL:

$$
\begin{aligned}
Y_{t}= & h\left(X_{T}\right)+\int_{t}^{T} f\left(s, X_{s^{-}}, Y_{s^{-}}, Z_{s}\right) d s+\int_{t}^{T} g\left(s, X_{s^{-}}, Y_{s^{-}}\right) d|\eta|_{s} \\
& -\int_{t}^{T} Z_{s} d W_{s}-\int_{t}^{T} U_{s} d\left(N_{s}-\lambda s\right)
\end{aligned}
$$

has a unique solution

$$
\left(Y_{t}, Z_{t}, U_{t}\right)=\left(u\left(t, X_{t}\right), \sigma\left(X_{t}\right) \partial_{x} u\left(t, X_{t}\right), u\left(t, X_{t^{-}}+c\left(X_{t^{-}}\right)\right)-u\left(t, X_{t^{-}}\right)\right),
$$

where $u$ is solution of the PDIE:

$$
\begin{gathered}
\partial_{t} u(t, x)-\lambda c(x) \partial_{x} u(t, x)+\frac{1}{2} \sigma^{2}(x) \partial_{x x}^{2} u(t, x)+u(t, x+c(x))-u(t, x) \\
+f\left(t, x, u(t, x), \sigma(x) \partial_{x} u(t, x)\right)=0 \quad \forall(t, x) \in[0, T) \times \Theta \\
n(x) \partial_{x} u(t, x)+g(t, x, u(t, x))=0 \quad \forall(t, x) \in[0, T) \times\{-l ; l\} \\
u(T, x)=h(x) \quad \forall x \in \bar{\Theta}
\end{gathered}
$$

\section{References}

[1] K. Bahlali, M. Eddahbi, and E. Essaky, BSDE associated with Lévy processes and application to PDIE, Journal of Applied Mathematics and Stochastic Analysis 16 (2003), no. 1, 1-17.

[2] K. Bahlali and E. Pardoux, Backward stochastic differential equations with locally monotone coefficient, preprint, 2001.

[3] G. Barles, R. Buckdahn, and E. Pardoux, Backward stochastic differential equations and integralpartial differential equations, Stochastics and Stochastics Reports 60 (1997), no. 1-2, 57-83.

[4] J.-M. Bismut, Conjugate convex functions in optimal stochastic control, Journal of Mathematical Analysis and Applications 44 (1973), 384-404.

[5] B. Boufoussi and Y. Ouknine, On a SDE driven by a fractional Brownian motion and with monotone drift, Electronic Communications in Probability 8 (2003), 122-134.

[6] Ph. Briand and R. Carmona, BSDEs with polynomial growth generators, Journal of Applied Mathematics and Stochastic Analysis 13 (2000), no. 3, 207-238.

[7] J. Cvitanić and I. Karatzas, Backward stochastic differential equations with reflection and Dynkin games, The Annals of Probability 24 (1996), no. 4, 2024-2056.

[8] R. W. R. Darling and E. Pardoux, Backwards SDE with random terminal time and applications to semilinear elliptic PDE, The Annals of Probability 25 (1997), no. 3, 1135-1159.

[9] N. El Karoui, S. Peng, and M. C. Quenez, Backward stochastic differential equations in finance, Mathematical Finance 7 (1997), no. 1, 1-71.

[10] N. El-Karoui and S. Hamadène, BSDEs and risk-sensitive control, zero-sum and nonzero-sum game problems of stochastic functional differential equations, Stochastic Processes and Their Applications 107 (2003), no. 1, 145-169.

[11] S. Hamadène and J.-P. Lepeltier, Zero-sum stochastic differential games and backward equations, Systems \& Control Letters 24 (1995), no. 4, 259-263.

[12] M. Kobylanski, Résultats d'existence et d'unicité pour des équations différentielles stochastiques rétrogrades avec des générateurs à croissance quadratique, Comptes Rendus de l'Académie des Sciences. Série I. Mathématique 324 (1997), no. 1, 81-86.

[13] J. P. Lepeltier and J. San Martin, Backward stochastic differential equations with continuous coeffcient, Statistics \& Probability Letters 32 (1997), no. 4, 425-430. 
[14] A. Løkka, Martingale representation, chaos expansion and Clark-Ocone formulas, MPS-RR 199922, MaPhySto, 1999.

[15] J.-L. Menaldi and M. Robin, Reflected diffusion processes with jumps, The Annals of Probability 13 (1985), no. 2, 319-341.

[16] D. Nualart and W. Schoutens, Chaotic and predictable representations for Lévy processes, Stochastic Processes and Their Applications 90 (2000), no. 1, 109-122.

[17] _ Backward stochastic differential equations and Feynman-Kac formula for Lévy processes, with applications in finance, Bernoulli 7 (2001), no. 5, 761-776.

[18] E. Pardoux, Backward stochastic differential equations and viscosity solutions of systems of semilinear parabolic and elliptic PDEs of second order, Stochastic Analysis and Related Topics, VI (Geilo, 1996), Progr. Probab., vol. 42, Birkhäuser Boston, Massachusetts, 1998, pp. 79-127.

[19] _ BSDEs, weak convergence and homogenization of semilinear PDEs, Nonlinear Analysis, Differential Equations and Control (Montreal, QC, 1998), NATO Sci. Ser. C Math. Phys. Sci., vol. 528, Kluwer Academic, Dordrecht, 1999, pp. 503-549.

[20] E. Pardoux and S. G. Peng, Adapted solution of a backward stochastic differential equation, Systems \& Control Letters 14 (1990), no. 1, 55-61.

[21] E. Pardoux and S. Zhang, Generalized BSDEs and nonlinear Neumann boundary value problems, Probability Theory and Related Fields 110 (1998), no. 4, 535-558.

[22] S. G. Peng, Probabilistic interpretation for systems of quasilinear parabolic partial differential equations, Stochastics and Stochastics Reports 37 (1991), no. 1-2, 61-74.

[23] P. E. Protter, Stochastic Integration and Differential Equations, 2nd ed., Stochastic Modeling and Applied Probability, Springer, Berlin, 2005, Version 2.1.

Mohamed El Otmani: Department of Mathematics, Faculty of Sciences Semlalia,

Cadi Ayyad University, Marrakesh, BP 2390, Morocco

E-mail address:m.elotmani@ucam.ac.ma 


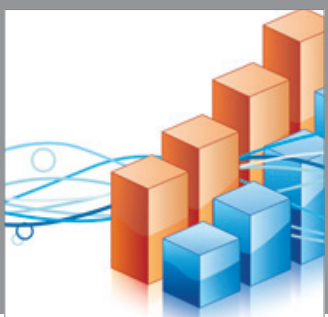

Advances in

Operations Research

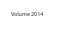

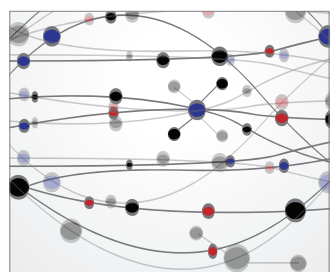

\section{The Scientific} World Journal
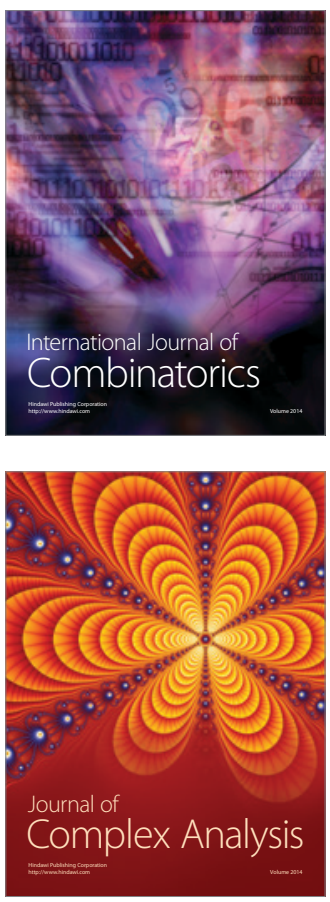

International Journal of

Mathematics and

Mathematical

Sciences
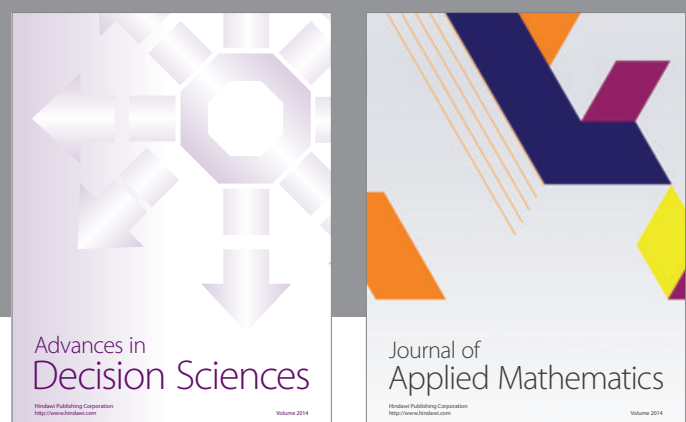

Journal of

Applied Mathematics
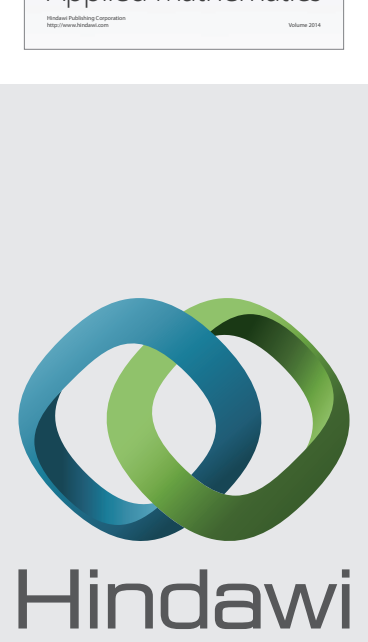

Submit your manuscripts at http://www.hindawi.com
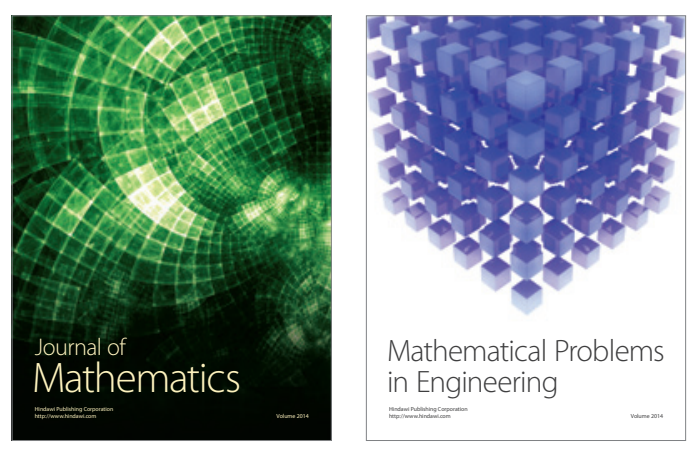

Mathematical Problems in Engineering
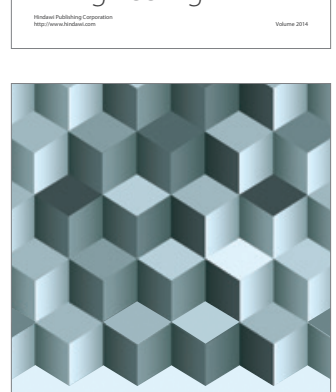

Journal of

Function Spaces
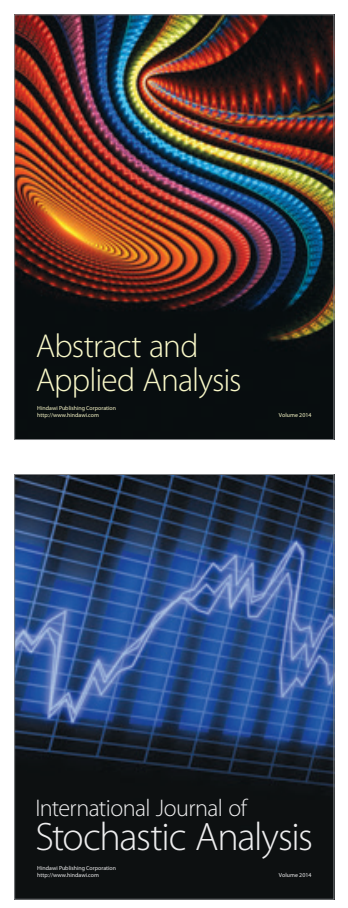

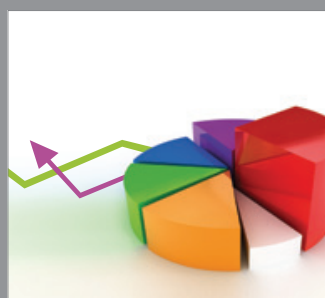

ournal of

Probability and Statistics

Promensencen
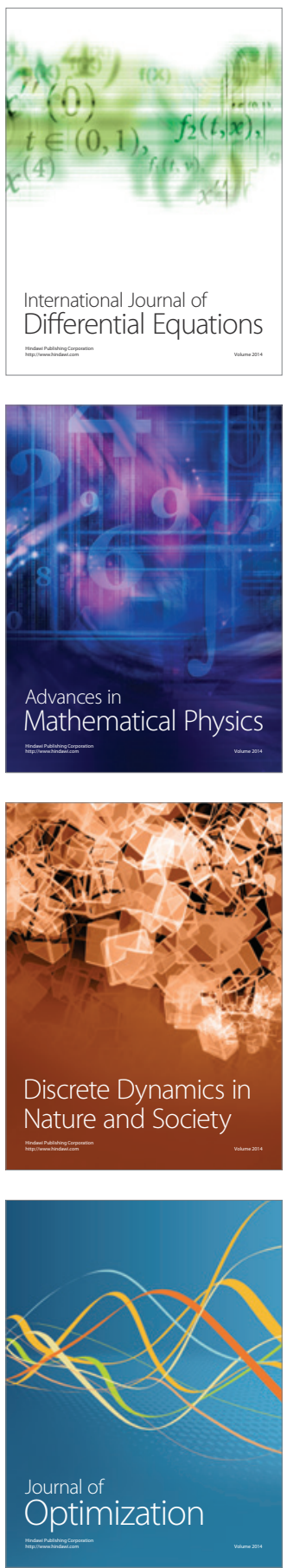